\title{
Mediation and Moderation in Statistical Network Models
}

\author{
Scott Duxbury \\ PhD Candidate in Sociology \\ The Ohio State University \\ email: duxbury.5@osu.edu \\ July 17, 2019
}

\begin{abstract}
Statistical network methods have grown increasingly popular in the social sciences. However, like other nonlinear probability models, statistical network model parameters can only be identified to a scale and cannot be compared between groups or models fit to the same network. This study addresses these issues by developing methods for mediation and moderation analyses in exponential random graph models (ERGM). It first discusses ERGM as an autologistic regression to illustrate that ERGM estimates can be affected by unobserved heterogeneity. Second, it develops methods for mediation analysis for both discrete and continuous mediators. Third, it provides recommendations and methods for interpreting interactions in ERGM. Finally, it considers scenarios where interactions are implicated in mediation analysis. The methodological discussion is accompanied with empirical applications and extensions to other classes of statistical network models are discussed.
\end{abstract}


Statistical network models have grown increasingly popular in the social sciences. Advances in statistical network modeling over the past 15 years have been credited with moving social network studies beyond descriptions of local and global structure into the realm of inferential analysis (Snijders, 2011). Unlike generalized linear models (GLM), which assume independent and identically distributed data, statistical network models possess the unique ability to obtain unbiased estimates for endogenous network effects. The ability to parse out endogeneity in graph statistics allows researchers to evaluate widely-cited theoretical models of the generative properties of social networks (e.g., Coleman, 1990; Granovetter, 1973). Indeed, statistical network models have been applied to test theories of racial boundaries (Wimmer and Lewis, 2010), gang violence (Papachristos, Hureau, and Braga, 2013), and the influence of cultural tastes on network selection (Lewis and Kaufman, 2018).

Despite the growing popularity of statistical network models in the social sciences, methodologies for parameter inference have not kept pace with model development. Particularly, parameters cannot be compared between groups or models fit to the same network. Because the most popular statistical network models are nonlinear probability models (see Frank and Strauss, 1986; Snijders, 2001), ${ }^{1}$ parameters are only identifiable to a scale. When the scale changes between models fit to the same data, differences in coefficients cannot be compared between models or interpreted as evidence of mediation (Hayes and Preacher, 2010; Mackinnon and Dwyer, 1993). When the scale is different between groups, coefficients for interactions and their $z$-statistics are often biased and cannot be interpreted as evidence of moderation (Allison, 1999; Long and Mustillo, 2018; Mustillo, Landerman, and Land, 2012; Williams, 2009). While these issues are well-known in GLM (see Allison, 1999; Mackinnon and Dwyer, 1993), methods for mediation and moderation analyses have yet to be developed for statistical network models. Consequently, conclusions in the current research literature related to indirect pathways and interactions have been reached without proper assessment.

Although methods have not been developed for mediation and moderation analysis in

\footnotetext{
${ }^{1}$ An example of a linear statistical network model is the network autocorrelation model applied to a continuous outcome.
} 
statistical network models, network scholars are often interested in assessing mediation and moderation. Researchers typically compare coefficients between models to determine whether a variable of interest has an indirect effect on an outcome through some mediating pathway or whether including a variable changes the strength of an empirical association. Examples in the applied literature include assessing whether endogenous network processes explain the effect of some exogenous covariate, like spatial proximity (Papachristos and Bastomski, 2018; Papachristos et al., 2013) or homophily (Goodreau, Kitts, and Morris, 2009). Likewise, between group comparisons are of interest when researchers want to assess whether the effect of a variable differs across levels of a moderator. A widely cited example is selective sorting in mating and friendship networks, where a potential friend or mate is more appealing if they are similar to a person on certain characteristics, like race or income (Lewis, 2013; McPherson, Smith-Lovin, and Cook, 2001; Wimmer and Lewis, 2010).

This study develops methods for assessing mediation and moderation in statistical network models with a focus on exponential random graph models (ERGM), though the methods proposed here can be extended to any parametric statistical network model with valid predictions. The first section casts ERGM as a nonlinear probability model and introduces the problem of unobserved heterogeneity. The second section develops strategies for mediation analysis in ERGM. In the third section, the problem of moderation is outlined and methods are proposed for improving the interpretation of interactions. The fourth section examines special cases when an interaction is either a direct effect or mediator in mediation analyses. The methods are applied to revisit the question of whether friendships form due to shared attributes or triadic structure (e.g., Goodreau et al., 2009). ${ }^{2}$

\section{Unobserved Heterogeneity in ERGM}

ERGMs are a class of statistical network models that represent network (graph) probabilities with the exponential family random graph distribution. Common ERGM formulations are

\footnotetext{
${ }^{2}$ Reproducible R code to carry out the empirical examples is provided in the Supplementary Materials.
} 
Erdos-Renyi models (Erdos and Renyi, 1959), dyad independence ( $p 1$ ) models (Holland and Leinhardt, 1981), Markov graph models (Frank and Strauss, 1986), and curved-family models (Hunter, 2007; Snijders, Pattison, Robins, and Handcock, 2006). In most applications, ERGMs are used to represent some kind of dyadic dependence structure (e.g., Markov or curved ERGMs) when modeling tie probabilities in binary cross-sectional networks, though extensions to dynamic and weighted networks exist (Desmarais and Cranmer, 2012b; Hanneke, Fu, and Xing, 2010; Krivitsky, 2012).

Most current implementations represent ERGM with the joint form of the model (Besag, 1974; Frank and Strauss, 1986; Geyer and Thompson, 1992; Snijders, 2002). Given a network $Y$ with $y_{i j}$ ties connecting actors $i$ and $j$, ERGM estimates the probability of observing $Y$ as a function of exogenous actor level characteristics $x$ and sufficient graph statistics $z(y)$. The joint form of an ERGM has the following probability mass function:

$$
\operatorname{Pr}(Y=y \mid z(y), x)=\frac{\exp \left(\theta^{T}(z(y), x)\right)}{\kappa(\theta)},
$$

where $\theta$ is the parameter vector and $\kappa(\theta)=\sum \exp \left(\theta^{T}(z(y), x)\right)^{\prime}$ is a normalizing constant representing all possible network configurations for a network of the same size. Due to the intractability of $\kappa(\theta)$ in most networks of interest, the denominator is typically approximated using Markov Chain Monte Carlo (MCMC) sampling (Geyer and Thompson, 1992; Snijders, 2002), where a large distribution of possible networks are simulated and randomly sampled to asymptotically approximate the covariance matrix. ${ }^{3}$ If the Markov chain has reached equilibrium, the MCMC likelihood estimator is equivalent to the likelihood estimator for the conditional tie probabilities. Parameters are thus interpretable as the increase/decrease in log-odds of an $i j$ tie given a one unit change in a focal covariate effect. ${ }^{4}$

\footnotetext{
${ }^{3}$ This is the Geyer-Thompson approach, which uses a Metropolis-Hastings algorithm and is the most widely applied strategy. Another common estimation algorithm is stochastic approximation (Snijders, 2002), which uses smaller random MCMC samples and more frequent updating.

${ }^{4}$ The endogenous ERGM parameters corresponding to $z(y)$ also have a macro-level interpretation, but this is rarely used in applied sociological research. I refer the reader to Block, Stadtfeld, and Snijders (2019) or Lusher, Koskinen, and Robins (2013) for a discussion of the macro-level properties of ERGMs.
} 
Perhaps because most ERGMs are estimated using the joint form of the model, ERGM is less frequently discussed as a nonlinear probability model than it was when the statistical properties of ERGM were first derived (see Holland and Leinhardt, 1981; Wasserman and Patterson, 1996). A well-known issue in nonlinear probability models is unobserved heterogeneity, where coefficients can only be identified to a scale. Unobserved heterogeneity renders coefficients non-comparable between models fit to the same data (Karlson, Holm, and Breen, 2012; Mackinnon and Dwyer, 1993; Winship and Mare, 1983) and biases interaction coefficients (Allison, 1999; Mood, 2010). These issues are also present in ERGM but typically go unaddressed.

Consider the tie variable $y_{i j}$, where $y_{i j}=1$ if $i$ and $j$ are connected and $y_{i j}=0$ otherwise. ERGM can be written as an autologistic regression using the conditional form of the model (Holland and Leinhardt, 1981; Strauss and Ikeda, 1990),

$$
\frac{\hat{p}_{i j}}{1-\hat{p}_{i j}}=\frac{\operatorname{Pr}\left(Y_{i j}=1 \mid X=x, Y_{-i j}=y_{i j}\right)}{\operatorname{Pr}\left(Y_{i j}=0 \mid X=x, Y_{-i j}=y_{-i j}\right)}=\exp \left(\theta_{\text {endogenous }}^{T} \delta_{i j}^{+}(y)+\theta_{\text {exogenous }}^{T}\left(x_{i j}\right)\right),
$$

with conditional tie probability

$$
\hat{p}_{i j}=\frac{\exp \left(\theta_{\text {endogenous }}^{T} \delta_{i j}^{+}(y)+\theta_{\text {exogenous }}^{T}\left(x_{i j}\right)\right)}{1+\exp \left(\theta_{\text {endogenous }}^{T} \delta_{i j}^{+}(y)+\theta_{\text {exogenous }}^{T}\left(x_{i j}\right)\right)},
$$

where $\delta_{i j}^{+}$is the change in parameterized graph statistic when a focal tie $y_{i j}$ is toggled from 0 to 1 . Autologistic regression earns its name by employing the same functional form as a logistic regression (Besag, 1972), but is distinct from logistic regression in that tie variables are treated as conditional on the entire graph structure and are not assumed to be independent. ${ }^{5}$ A large statistical and methodological literature details the inferential difficulties that arise when using a logistic functional form to model latent probabilities

\footnotetext{
${ }^{5}$ Special dyad independent cases of ERGM reduce to logistic regression (Holland and Leinhardt, 1981), and some ERGMs are estimated as Firth models or logistic mixed models (Box-Steffensmeier, Christenson, and Morgan, 2018; van Duijn, Gile, and Handcock, 2009; van Duijn, Snijders, and Zijlstra, 2004). These models are infrequently used in practice, however, as pseudolikelihood and penalized pseudolikelihood estimates suffer from known biases in models with complex dependence structure.
} 
(Allison, 1999; Cramer, 2007; Karlson et al., 2012; Mackinnon and Dwyer, 1993; Mood, 2010).

Because tie probabilities are not observed directly, they are predicted with unknown error. Following convention in the literature on logistic regression (Hosmer and Lemeshow, 2000; Long, 1997), we can write the latent data generating model as a function of model terms. For the purposes of simplicity, we use the more compact notation $X$ to denote all model terms, whether endogenous change statistics or exogenous covariate effects. The latent model can be written,

$$
Y^{*}=\alpha^{T} X+\tau \epsilon
$$

where $Y^{*}$ is the latent propensity of observing an $i j$ tie that is greater than zero if $y_{i j}=1$ and is less than or equal to zero if $y_{i j}=0 . \alpha$ is the latent parameter vector and $\epsilon$ is the latent error. Since $\epsilon$ is unmeasured and unknown, we must assume some distribution for the error or the model cannot be estimated. In logistic probability models, we assume that $\epsilon$ is a logistic random variable with a mean of zero and a fixed variance of $\frac{\pi^{2}}{3} \approx 3.29$. The scale $\tau$ relates the assumed logistic distribution of $\epsilon$ to the true error distribution of the latent linear model.

The invariance of $\epsilon$ is the cause of problems related to unobserved heterogeneity in ERGM. To see this, we can write the equivalence of the latent and observed models as (see Allison, 1999):

$$
\theta^{T} X=\frac{\alpha^{T} X}{\tau}
$$

and the equivalence of model parameters as: $\theta=\alpha / \tau$. Because $\epsilon$ is invariant, the inclusion of a new model term changes the value of $\tau$ and, by extension, the values of $\theta$. When the new model term is a strong predictor, the change in $\tau$ will typically be substantial (Karlson et al., 2012). We can obtain an equivalent result for any network model where the error distribution is both unknown and invariant. Unobserved heterogeneity is therefore a problem in any statistical network model that can be represented as a nonlinear probability 
model. This includes stochastic actor-oriented models (SAOM), relational event models, dynamic actor-event models, and both dynamic and generalized ERGMs.

Since we do not know the value of $\tau$, we cannot determine whether change in coefficient size and significance between models and groups results from mediation, moderation, or unobserved heterogeneity. Unobserved heterogeneity has been the topic of sustained research programs for mediation and moderation analyses in nonlinear probability models (Allison, 1999; Hayes and Preacher, 2010; Long and Mustillo, 2018; Mackinnon and Dwyer, 1993; Mustillo et al., 2012; Williams, 2009; Winship and Mare, 1983). However these issues have yet to be addressed in network analysis.

\section{Mediation}

Network analysts are often interested in mediation when they seek to identify through what pathways network processes influence various outcomes. Researchers may also conduct mediation analysis when they are interested in quanitfying the degree of confounding between two variables. Common examples include when researchers want to identify whether network processes explain the direct effect of some actor-level, dyadic, or spatial phenomenon. For instance, Schaefer, Kornienko, and Fox (2011) used SAOM to assess whether the mechanism that explains the positive effect of depression homophily on tie probabilities in adolescent friendship networks is preferential attachment, avoidance, or withdrawal. Melamed, Harrell, and Simpson (2018) conducted an experiment to assess whether network dynamics promote cooperation in economic games directly by excluding defectors or indirectly by conferring reputation. Papachristos et al. (2013) applied ERGM to assess whether transitive structures explain the positive effect of spatial proximity on shootings in a gang violence network.

Four pathways are involved in mediation analysis (see Baron and Kenny, 1986). These are displayed in the directed acyclic graph in Figure 1, where $X$ is a direct effect or antecedent, ${ }^{6}$

\footnotetext{
${ }^{6}$ In the literature on mediation analysis, $X$ is often referred to as an exogenous variable and $M$ as endogenous to the $X$ pathway. I avoid this language to reduce confusion with the use of exogenous and
} 
Figure 1: Pathways for mediation analysis.

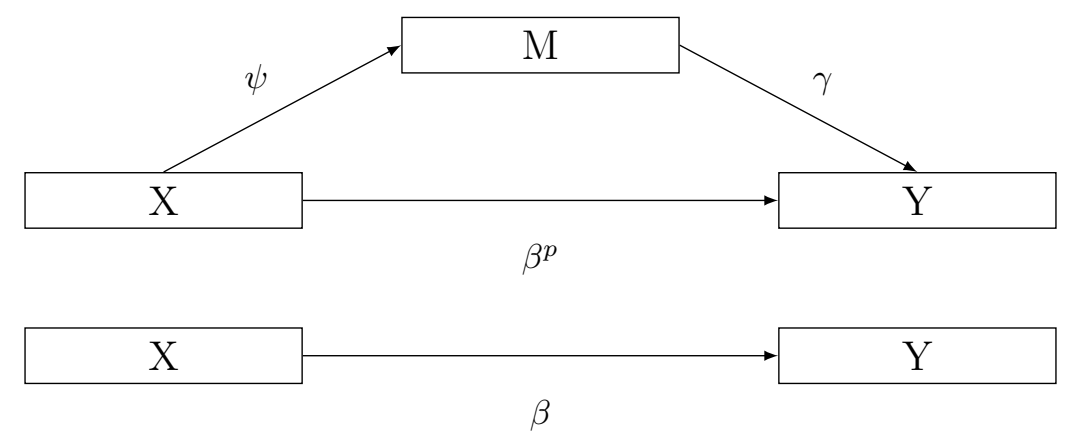

$Y$ is an outcome variable, and $M$ is the mediator. $\beta$ is the total effect of $X$ on $Y$. The cyclical figure decomposes $\beta$ into its composite parts, where $\beta^{p}$ is the partial or direct effect of $X$ on $Y, \psi$ is the effect of $X$ on $M$, and $\gamma$ is the effect of $M$ on $Y$. The indirect effect of $X$ on $Y$ is the portion of $\beta$ which is explained by $M$. The indirect effect is traditionally measured using either the difference in coefficients $\beta-\beta^{p}$ (Judd and Kenny, 1981), or the product of coefficients $\psi \gamma$ (Sobel, 1986). While the two calculations are equivalent in linear models (Mackinnon, Warsi, and Dwyer, 1995), the product of coefficients is more frequently used in practice because unobserved heterogeneity problematizes the difference in coefficients in nonlinear probability models (Mackinnon and Dwyer, 1993; Mackinnon, Lockwood, Brown, and Wang, 2007).

\subsection{Problems posed by network data}

Network data present important difficulties for measuring the indirect effect. The most common approach for assessing mediation in ERGM is to use the differences in coefficients. In the first model, an ERGM is specified without $M$. In the second model, $M$ is included. If the parameter for $X$ changes in size or significance, it is interpreted as mediation. However, change in significance, in itself, is not necessarily statistically significant (Gelman and Stern, 2006). Because differences in coefficient size can result from variation in $\tau$, the change in coefficient size is cannot be attributed to mediation or confounding.

endogenous in network analysis, where an endogenous variable refers to statistics computed on the graph structure. 
The product of coefficients is also usually unavailable. To obtain a point estimate for $\psi$, researchers typically regress $M$ on $X$ with a linear or generalized linear model, where the coefficient for $X$ is the point estimate for $\psi$. If a researcher does the same with network data the $\psi$ coefficient will typically be biased because GLMs are inappropriate for representing network data with non-trivial dependence structure. ${ }^{7}$ This is a particularly problematic issue because most current methods for mediation analysis either calculate the product of coefficients (Barrett, Cruz, and Lockhart, 2019; Breen, Karlson, and Holm, 2013; Hayes and Preacher, 2010; Mackinnon and Dwyer, 1993; Sobel, 1982; VanderWeele, 2011) or require a model of the $\psi$ pathway to compute the difference in coefficients (Imai, Tingley, and Keele, 2010; Karlson et al., 2012; Mackinnon et al., 2007).

\subsection{Comparisons of average marginal effects}

An alternative to comparing coefficients between models is to compare average marginal effects (AMEs). While coefficients can only be identified to a scale in nonlinear probability models, changes in $\tau$ do not affect expectations (Long, 1997; Long and Mustillo, 2018). ${ }^{8}$ The use of AMEs as a framework for comparing coefficients between nonlinear probability models fit to the same data has been discussed in several places (Barrett et al., 2019; Hayes and Preacher, 2010; Karlson et al., 2012; Wooldridge, 2002). The method can be applied to evaluate mediation with discrete and continuous mediators and to assess joint mediation with little modification. Moreover, since it relies on postestimation, it can be translated to any parametric statistical network model with valid predictions.

The marginal effect for a variable is the expected increase in tie probability when the variable increases by 1 . For a continuous variable, we define the marginal effect with respect

\footnotetext{
${ }^{7}$ If any variable is an endogenous graph statistic, GLM parameters will be biased because the model is not dyad independent. If no variable is an endogenous graph statistic, then GLM estimates will only be unbiased if the underlying data generating process is truly dyad independent. Otherwise, GLM parameters will suffer from omitted variable bias because of unmeasured structural effects, which biases even model parameters that are uncorrelated with the omitted variable in nonlinear probability models (Mood, 2010).

${ }^{8}$ Expectations can be affected by unobserved heterogeneity if the logistic formulation of the model is not a good representation of tie probabilities. However, Cramer (2007) shows that average marginal effects are remarkably robust even in these circumstances.
} 
to $X$ as its partial derivative,

$$
M E_{\theta_{x}}^{i j}=\theta_{x} \frac{\delta \hat{p}_{i j}}{\delta X_{i j}} .
$$

For binary variables, the partial derivative is equivalent to the difference in tie probabilities when $X$ changes from 0 to 1 . The superscript $i j$ indexes that all dyads in the ERGM sample space have a marginal effect.

The average marginal effect (AME) of $X$ is the mean marginal effect; that is, the average change in tie probability given a one unit increase in $X$ :

$$
A M E_{\theta_{x}}=\theta_{x} \frac{1}{n} \Sigma_{i j=1} \frac{\delta \hat{p}_{i j}}{\delta X_{i j}}=\frac{\Sigma_{i j=1} M E_{\theta_{x}}^{i j}}{n}
$$

where $n$ is the number of dyads in the ERGM sample space. In linear models, the AMEs are equivalent to regression coefficients. Standard errors are obtained with the Delta method (see Agresti, 2002). The covariance matrix for the AMEs is the crossproduct $D_{\theta} \hat{\Omega}_{\theta} D_{\theta}^{T}$, where $D_{\theta}$ is the Jacobian matrix or gradient of the AMEs and $\hat{\Omega}_{\theta}$ is the covariance matrix of the ERGM estimator.

Since the $\psi$ pathway is usually unavailable in ERGM, we compute the indirect effect from the difference in AMEs. We define the two models required for mediation analysis as nested ERGMs:

$$
\begin{aligned}
& 1: \log \left(\frac{\hat{p}}{1-\hat{p}}\right)=\theta_{x} X+\theta_{z}^{T} Z \\
& 2: \log \left(\frac{\hat{p}}{1-\hat{p}}\right)=\theta_{x}^{p} X+\theta_{m} M+\theta_{z}^{T} Z,
\end{aligned}
$$

where $Z$ is a set of controls. The indirect effect is the change in AME between models:

$$
A M E_{\theta_{x}}^{\text {indirect }}=A M E_{\theta_{x}}-A M E_{\theta_{x}^{p}}
$$

The interpretation for the indirect effect is the average marginal effect of $X$ explained by 
$M$, or the average change in tie probability indirectly attributable to the direct effect through a mediating pathway. To assess significance, we test the null hypothesis $H_{0}: A M E_{\theta_{x}}^{\text {indirect }}=0$. An appealing property of this measure is that $A M E_{\theta_{x}}$ is equal to the sum of the indirect and partial effects, which is not usually true in mediation analyses in nonlinear probability models (Mackinnon, 2008; Mackinnon et al., 2007). The total effect is $A M E_{\theta_{x}^{p}}+A M E_{\theta_{x}}^{\text {indirect }}=$ $A M E_{\theta_{x}}$. The proportion mediated is $1-\frac{A M E_{\theta_{x}^{p}}}{A M E_{\theta_{x}}}$.

A common strategy for estimating the standard error for the indirect effect is to use bootstrap resampling (Barrett et al., 2019; Bollen and Stine, 1990; Hayes and Preacher, 2010), which involves sampling from the data, re-estimating the model, and calculating the indirect AME a very large number of times. Since model run-times are often restrictive in ERGM, bootstrapping will scale poorly to large networks, high dimensional models, and curved ERGMs with complex dependence structures. Instead, Delta standard errors can be calculated for the indirect effect. We obtain the standard error for the indirect effect using the Variance Sum Law:

$$
\sigma\left(A M E_{\theta_{x}}^{\text {indirect }}\right)=\sqrt{\operatorname{Var}\left(A M E_{\theta_{x}}\right)+\operatorname{Var}\left(A M E_{\theta_{x}^{p}}\right)-2 \operatorname{Cov}\left(A M E_{\theta_{x}}, A M E_{\theta_{x}^{p}}\right)},
$$

where the variances are Delta method estimates. Because the standard errors for $\theta_{x}$ and $\theta_{x}^{p}$ are obtained from distinct covariance matrices, we cannot use the crossproduct of the Jacobian matrix to obtain the covariance between $A M E_{\theta_{x}}$ and $A M E_{\theta_{x}^{p}}$. Instead, we calculate the covariance from the correlation between marginal effects and the Delta standard errors for each AME: $\operatorname{Cov}\left(A M E_{\theta_{x}}, A M E_{\theta_{x}^{p}}\right)=\operatorname{Corr}\left(M E_{\theta_{x}}^{i j}, M E_{\theta_{x}^{p}}^{i j}\right) \cdot \sigma\left(A M E_{\theta_{x}}\right) \cdot \sigma\left(A M E_{\theta_{x}^{p}}\right)$. Since the difference in coefficients is equivalent to the product of coefficients and AMEs are equivalent to coefficients in linear regression, this method reduces to a Sobel test if applied to a linear model (see Sobel, 1982). It can therefore be regarded as Sobel-type test of the indirect effect. $^{9}$

\footnotetext{
${ }^{9}$ The Sobel test has been critiqued for providing conservative standard errors in small sample spaces with fewer than roughly 200 observations, where the assumption of asymptotic normality is often violated (Bollen and Stine, 1990; Mackinnon, 2008; Mackinnon, Lockwood, and Williams, 2004). Since the dyad sample space
} 
An appealing property of mediation using AMEs is that it has direct applications to joint mediation (Preacher and Hayes, 2008; VanderWeele and Vansteelandt, 2013). Joint mediation exists when multiple mediators explain a direct effect in conjunction. Because the indirect effect is calculated from the difference in AMEs, no additional considerations are necessary to assess joint mediation. A researcher simply includes multiple mediating variables into the full model, instead of a single mediator, and calculates the difference in AMEs. The indirect AME is the joint indirect effect. As will be discussed in detail shortly, joint mediation is particularly informative when $M$ is endogenous.

The assumptions of mediation analysis in ERGM are identical to traditional mediation analyses (see Mackinnon, 2008). It is assumed that there are no omitted confounders correlated with both $M$ and $X$, that the functional (logistic) form of the models is appropriate, and that all covariates have been accurately measured. It is also prudent to emphasize the implicit assumption that both ERGMs converge (e.g., the estimates for both models are obtained from the stationary MCMC distribution and the MC-MLE is identified).

\subsection{Example 1: Revisiting Birds of a Feather or Friend of a Friend?}

Social networks are typified by high levels of local clustering (Newman, 2010; Watts and Strogatz, 1998). Two explanations are generally advanced for this high level of clustering. The homophily hypothesis posits that social ties are more likely to form if actors incident to the tie in question are similar on a variety of characteristics (McPherson et al., 2001). This is typically elaborated as a preferential attachment mechanism, where actors prefer ties with similar alters, though Schaefer et al. (2011) provide compelling evidence for alternative mechanisms that generate depression homophily in friendship networks. The second hypothesis is structural balance (Granovetter, 1973). This hypothesis states that proximity in social space--usually formulated as triad closure-facilitates tie formation. The homophily

is usually quite large in ERGM, it is reasonable to assume asymptotic normality for most applications. For instance, even a small undirected network of 20 vertices has $\frac{20 \cdot(20-1)}{2}=190$ dyads. In networks with 20 or fewer vertices, bootstrap standard errors are a pragmatic alternative. 
and triad closure hypotheses are rarely construed as competing. For instance, Goodreau et al. (2009) use ERGM to study triad closure and selective mixing in a pooled sample of 59 school networks, finding that including a triad closure coefficient tends to reduce the size of homophily coefficients by 5 to $15 \%$ (p. 115). However, because of unobserved heterogeneity, we cannot be certain that these differences in coefficient size are attributable to real social processes.

We revisit this example using the Faux Mesa High network available via the statnet package for R (see Handcock, Hunter, Butts, Goodreau, and Morris, 2008, for details). The network is undirected, entailing the friendship ties between students of a simulated social network based on one in-school network in the Longitudinal Study of Adolescent Health. The probability of tie formation is modeled as a function of students' grade, race, and sex. To assess whether triad structure mediates homophily, we also include grade, sex, and race homophily as matched nodal characteristics. Triad closure is measured as geometrically weighted edgewise shared partnerships (GWESP) with a fixed decay term of .5 (Hunter, 2007).

Model 1 specifies the model without the GWESP term (Table 1). The positive coefficient for students' grade indicates that older students and students in higher grades tend to have more friends, reflecting greater exposure to the school network. The negative coefficients for all racial categories in comparison to black students indicate that black students have the highest probability of forging friendships. The negative coefficient for male students indicates that male students tend to have fewer friends than female students. Turning to homophily, there is a preference towards same sex, same race, and same grade friendships.

Model 2 includes the GWESP term. The positive coefficient indicates that students have greater odds of becoming friends if they already share a mutual friend. There is also notable change in several coefficients. The coefficient for same grade friendships declines by $30 \%$. Similarly, the coefficients for same sex and same race friendships also decrease by $14 \%$ and $12 \%$ respectively. However, multiple other coefficients also decline in size. It is 
Table 1: ERGM of friendships in Faux Mesa High.

\begin{tabular}{lll}
\hline Parameters & Model 1 & Model 2 \\
\hline Edges & $-5.45^{* * *}(.70)$ & $-6.41^{* * *}(.41)$ \\
Grade & $.09^{* *}(.03)$ & $.07^{* * *}(.01)$ \\
Race (black is referent) & & \\
$\quad$ Latino & $-1.58^{* * *}(.24)$ & $-.98^{* * *}(.17)$ \\
$\quad$ N. American & $-1.13^{* * *}(.24)$ & $-.74^{* * *}(.16)$ \\
$\quad$ White & $-.86^{* * *}(.26)$ & $-.53^{* *}(.16)$ \\
$\quad$ Other & $-3.20^{* *}(1.02)$ & $-1.98^{*}(.90)$ \\
Male & $-.35^{* * *}(.09)$ & $-.10(.06)$ \\
Grade homophily & $3.00^{* * *}(.18)$ & $2.14^{* * *}(.16)$ \\
Sex homophily & $.64^{* * *}(.15)$ & $.55^{* * *}(.14)$ \\
Race homophily & $.83^{* * *}(.16)$ & $.73^{* * *}(.13)$ \\
GWESP & & $1.31^{* * *}(.09)$ \\
\hline AIC & 1,846 & 1,667 \\
BIC & 1,926 & 1,755 \\
\hline$* * * p<0.001,{ }^{* *} p<0.01,{ }^{*} p<0.05$. &
\end{tabular}

unclear whether the change in coefficient size can be attributed to mediation or unobserved heterogeneity.

Table 2 presents results from mediation analyses. The indirect effects for race and gender homophily are both small in size and insignificant. In contrast, $42.9 \%$ of the AME of grade homophily can be attributed to triad structure (proportion mediated $=.429$ ). The partial AME indicates that students are $1.6 \%$ more likely to forge friendships with students in the same grade than students in different grades $\left(A M E_{\text {Grade-match }}^{\text {partial }}=.016\right)$, while the indirect AME predicts an additional indirect .012 increase in tie probability for same-grade students due to triad formation $\left(A M E_{\text {Grade-match }}^{\text {indirect }}=.012\right)$. In total, students who share a grade are $2.8 \%$ more likely to be friends than students in different grades $\left(A M E_{\text {Grade-match }}^{\text {total }}=.028\right)$.

Results illustrate that triadic structure can explain roughly $43 \%$ of the effect of grade similarity, but there is little evidence that it explains the effect of sex or race homophily in the Faux Mesa High friendship network. However, as will be illustrated in the empirical examples to follow, additional considerations are needed when examining homophily and endogenous graph statistics involved in a mediation analysis. We will return to this example as these methods are introduced. 
Table 2: Marginal mediation analyses examining indirect AME for GWESP. Delta standard errors in parentheses.

\begin{tabular}{lllll}
\hline & Total AME & Direct AME & Indirect AME & Percent mediated \\
\hline Grade homophily & $.028^{* * *}(.002)$ & $.016^{* * *}(.002)$ & $.012^{* * *}(.002)$ & 42.9 \\
Sex homophily & $.006^{* * *}(.001)$ & $.004^{* *}(.002)$ & $.002(.002)$ & 26.6 \\
Race homophily & $.008^{* * *}(.002)$ & $.006^{* * *}(.002)$ & $.002(.002)$ & 27.9 \\
\hline${ }^{* * *} p<0.001,{ }^{* *} p<0.01,{ }^{*} p<0.05$. & & &
\end{tabular}

\subsection{Summary}

Unobserved heterogeneity renders ERGM parameters uncomparable between models. This section proposed a Sobel-type test for calculating the indirect effect and its standard error in statistical network models. There are many appealing properties to the framework, including straightforward applications to both discrete and continuous mediators, multiple mediation, and interpretability. Special considerations are needed when the direct effect is an interaction or the mediator is an endogenous structural parameter. The following sections develop these points in more detail.

\section{Moderation}

Moderation refers to when the effect of a focal variable varies at different levels of a second variable (Baron and Kenny, 1986). Moderation is assessed using interaction terms, which are usually product terms between independent covariates, but can also include other functions of independent variables, such as absolute differences. There are two problems related to moderation in ERGM. First, lower-order measures are often not parameterized, meaning that the significance of interaction terms and endogenous network effects may result from omitted variables. Second, the interpretation for interactions is usually incorrect because researchers do not account for unobserved heterogeneity across levels of a moderator, which can bias coefficients and z-statistics.

To illustrate, I reviewed all articles published in the past 10 years in either American Sociological Review or American Journal of Sociology that used ERGM or SAOM. Of the 
10 articles identified, all 10 specified at least one triadic effect and 9 specified a node matching term. Yet, only 1 article correctly interpreted these terms as interactions and only 2 controlled for relevant lower order terms. None of the articles addressed unobserved heterogeneity. The following section discusses these issues in more detail and provides tests, recommendations, and corrections for assessing moderation in ERGM.

\subsection{Recognizing and controlling for lower order terms}

When social scientists study a network, they are often only indirectly interested in the properties of ties. Instead, research questions usually focus on the properties of the vertices (see Snijders, 1996): Do people become friends with similar alters (McPherson et al., 2001)? If people are embedded in local clusters, does it increase the probability that they will become friends (Goodreau et al., 2009)? If my friend is the victim of a gunshot, does it increase the likelihood that I will be shot as well (Papachristos, Wildeman, and Roberto, 2015)? For many researchers, it is unintuitive to think of ties as observations and, by extension, that interactions are properties of tie variables. The focus of this section is to draw attention to common mistakes in ERG modeling and to outline corrections. The issues discussed in this subsection will not be new to methodologists, but are worth highlighting for practitioners.

Recognizing Interactions: Consider racial homophily. If I am studying whether a person is more likely to be friends with a same-race alter, the conceptual model does not appear to imply moderation. If I were to model racial homophily from a survey, I would code the variable as a binary (or potentially a count) variable indicating whether the respondent lists

a friend of the same race. In contrast, when I model a network at the dyadic level, my observations are tie variables. I am no longer modeling whether a person has a same-race friend; I am modeling whether nodes at either end of a tie (two friends) share an attribute (are the same race). This reduces to a product term that takes on a value of 1 if both people are the same race, and 0 otherwise (Lusher et al., 2013; Morris, Handcock, and Hunter, 2008). 
Table 3: Common ERGM Interaction Terms. $x$ is a tie variable, $a$ and $b$ are nodal attributes.

\begin{tabular}{|c|c|c|}
\hline Term & Formula & Lower Order Terms \\
\hline \multicolumn{3}{|l|}{ Undirected } \\
\hline Node matching & $\sum_{i<j} x_{i j} a_{i} a_{j}$ & Node $(a)$ \\
\hline Node mixing & $\sum_{i<j} x_{i j} a_{i} b_{j}$ & Node $(a, b)$ \\
\hline Triangle & $\sum_{i, j} x_{i j} x_{i k} x_{j k}$ & Two-star \\
\hline \multicolumn{3}{|l|}{ Directed } \\
\hline Node matching & $\sum_{i<j} x_{i j} a_{i} a_{j}$ & Sender $(a)$, receiver $(a)$ \\
\hline Outgoing node mixing & $\sum_{i<j} x_{i j} a_{i} b_{j}$ & Sender $(a)$, receiver $(b)$ \\
\hline Incoming node mixing & $\sum_{i<j} x_{i j} b_{i} a_{j}$ & Sender $(b)$, receiver $(a)$ \\
\hline $\begin{array}{l}\text { Reciprocal node } \\
\text { matching }\end{array}$ & $\sum_{i<j} x_{i j} x_{j i} a_{i} a_{j}$ & $\begin{array}{l}\text { Sender }(a) \text {, receiver }(a), \\
\text { reciprocity, node matching }\end{array}$ \\
\hline $\begin{array}{l}\text { Reciprocal node } \\
\text { mixing }\end{array}$ & $\sum_{i<j} x_{i j} x_{j i} a_{i} b_{j}$ & $\begin{array}{l}\text { Sender }(a, b) \text {, receiver }(a, b) \text {, } \\
\text { reciprocity, incoming node } \\
\text { mixing, outgoing node mixing }\end{array}$ \\
\hline Three-cycle & $\sum_{i, j} x_{i j} x_{j k} x_{k i}$ & Two-path \\
\hline Transitive triplet & $\sum_{i, j} x_{i j} x_{i k} x_{k j}$ & $\begin{array}{l}\text { Two-path, in-two-star, } \\
\text { out-two-star }\end{array}$ \\
\hline
\end{tabular}

The main effect of the product term is the race of the actor on either end of the tie, which is a single term in an undirected network and includes two terms for sender and receiver effects in a directed network. If the main effects are not parameterized, it cannot be determined that the effect of race homophily stems from matched nodal characteristics, or whether members of a particular racial group have higher levels of outgoing or incoming tie activity. This same issue arises across a number of commonly used dyad-level ERGM terms, including node mixing, absolute differences between continuous nodal attributes, and reciprocated node matching or mixing coefficients in directed networks.

Accounting for Nesting: A closely related but more peculiar issue arises from nesting in 
network structures. Many higher-order graph structures manifest from lower-order graph statistics (Faust, 2007). The peculiarity of this issue is that graph structures can be characterized as endogenous interactions-that is, product terms involving the dependent variable. It is impossible for higher-order graph structures to change without changing the value of at least one lower-order graph structure. Consequently, omitting lower-order structures yields a similar problem as omitting a main effect when modeling an interaction.

Consider the simplest case of a triangle in an undirected network. The triangle exists if three vertices are all connected to one another by three unique ties. The triangle term can also be written as an interaction-a product term between the existence of a focal tie and a two-star, where one actor is connected to two other actors. If the focal tie closes the twostar, the triangle statistic takes a value of 1 , and otherwise is 0 . However, because closing a triangle also creates two new two-stars, we cannot interpret the triangle coefficient as the effect of triads if we do not also control for two-stars. These same issues arise in curved ERGMs, when examining higher order graph structures, and directed networks, where the interactions between nesting structures can grow quite complex (Block, 2015).

Recommendations: The correction to these issues is to change model specification to accomodate relevant main effects and lower-order graph structures. It is not possible to provide an exhaustive list of graph structures and their nesting terms here-over 100 unique graph structures have been defined in the ergm package for R (Morris et al., 2008). However, some provisional guidance can be offered. Table 3 presents a number of common dyadic and triadic ERGM terms and their lower-order graph structures. In some circumstances, the inclusion of lower-order terms can problematize model convergence due to collinearitytype problems (see Duxbury, 2018). A number of tuning parameters can be set to the MCMC algorithm to promote convergence, such as increasing MCMC sample size or using machine learning methods to approximate the MLE in complex model spaces (Hinton and Salakhutdinov, 2006; Krivitsky, 2017). The R syntax in the Supplementary Materials for the following empirical application provides an example of how to improve convergence in 
Table 4: ERGM of friendships in Faux Mesa High including GWDSP.

\begin{tabular}{lll}
\hline Parameters & Model 3 & Model 4 \\
\hline Edges & $-8.03^{* * *}(.98)$ & $-6.92^{* * *}(.96)$ \\
Grade & $.13^{* * *}(.03)$ & $.10^{* * *}(.03)$ \\
Race (black is referent) & & \\
$\quad$ Latino & $-1.12^{* *}(.38)$ & $-.98^{* *}(.38)$ \\
$\quad$ N. American & $-.93^{*}(.38)$ & $-.85^{*}(.37)$ \\
$\quad$ White & $-.74(.40)$ & $-.74(.41)$ \\
$\quad$ Other & $-2.29^{*}(1.04)$ & $-2.08^{*}(1.01)$ \\
Male & $-.20(.10)$ & $-.19(.10)$ \\
Grade homophily & $3.17^{* * *}(.23)$ & $2.53^{* * *}(.22)$ \\
Sex homophily & $.62^{* * *}(.18)$ & $.54^{* *}(.17)$ \\
Race homophily & $.73^{* * *}(.20)$ & $.62^{* * *}(.18)$ \\
GWDSP & $.26^{* * *}(.07)$ & $.01(.08)$ \\
GWESP & & $1.32^{* * *}(.11)$ \\
\hline AIC & $-2,406$ & $-2,526$ \\
BIC & $-2,318$ & $-2,431$ \\
\hline${ }^{* * *} p<0.001,{ }^{* *} p<0.01,{ }^{*} p<0.05$. &
\end{tabular}

the context of a Metropolis-Hastings algorithm.

\subsection{Example 2: Revisiting Friend of a Friend}

The mediation analysis in Example 1 only considered a triad term, but not a term for twostars. Thus, we encounter an identification problem: We cannot be sure whether GWESP mediates the effect of node matching, or whether this is primarily driven by two-stars. Moreover, since closing a triangle creates two two-stars, we must also account for the joint indirect effect of two-stars and GWESP. We now return to Faux Mesa High to investigate this mediating pathway in more detail.

Model 3 includes a geometrically weighted dyadwise shared partnership (GWDSP) term with a fixed decay parameter of 1 without the GWESP term (Table 4). The positive effect of GWDSP indicates that two-stars increase tie probability. To assess whether there are independent effects of two-stars and triads, Model 4 includes the GWESP term, yielding the full model accounting for nesting structure. GWDSP is no longer significant, while GWESP is again positively related to tie probabilities. 
Figure 2: Mediating effects of GWDSP and GWESP, indirect effect point estimates and 95\% confidence intervals.
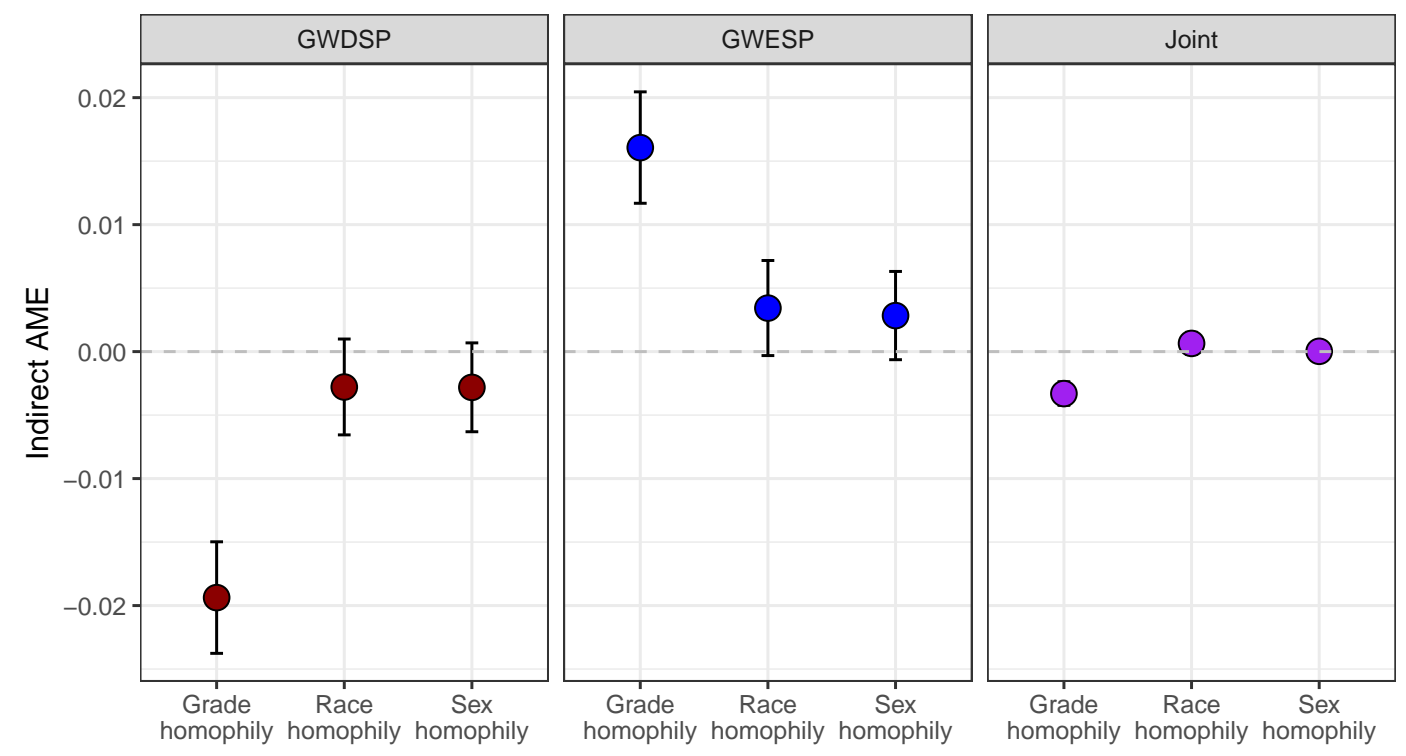

Figure 2 plots indirect effects from mediation analyses. There is a negative indirect effect when testing whether GWDSP mediates the effect of grade homophily on tie probabilities, but an insignificant indirect effect for both race and sex homophily. This indicates that grade homophily is negatively related to the formation of two-stars. The results for the mediating effect of GWESP on race, sex, and grade, homophily are consistent with Example 1, where only the indirect AME for grade homophily is positive and significant.

Since the GWESP and GWDSP pathways yield indirect effects with opposite signs, it is difficult to interpret the indirect effect for node matching. We can examine the pathway in more detail by assessing the mediating effect of GWESP on GWDSP and by assessing joint mediation. In fact, GWESP completely mediates the effect of GWDSP with a proportion mediated of $.97\left(A M E^{\text {indirect }}=.003, S E=.001, p<.001\right)$, indicating that the direct effect of GWDSP is approximately zero.

Table 5 reports results from joint mediation tests, and the indirect effects are plotted in Figure 2. Consistent with single mediator analysis, the indirect effects for both race and sex homophily are insignificant. An interesting result is that the joint indirect effect 
Table 5: Marginal mediation analyses examining joint indirect AME for GWESP and GWDSP. Delta standard errors in parentheses.

\begin{tabular}{lllll}
\hline & Total AME & Direct AME & Indirect AME & Percent mediated \\
\hline Grade homophily & $.016^{* * *}(.001)$ & $.019^{* * *}(.002)$ & $-.003^{* * *}(.000)$ & 21.1 \\
Sex homophily & $.004^{* * *}(.001)$ & $.004^{* *}(.001)$ & $.000(.000)$ & .6 \\
Race homophily & $.005^{* * *}(.001)$ & $.005^{* * *}(.001)$ & $.001(.000)$ & 12.2 \\
\hline${ }^{* * *} p<0.001,{ }^{* *} p<0.01,{ }^{*} p<0.05$. & & &
\end{tabular}

for GWESP and GWDSP is negative, indicating that the overall indirect effect of grade homophily is negative. This is because the negative effect of grade homophily on GWDSP $\left(A M E_{\text {grade-match } \rightarrow G W D S P}^{\text {indirect }}=-.019\right)$ is greater than the positive effect of grade homophily on GWESP (AME $\left.E_{\text {grade-match } \rightarrow G W E S P}^{\text {indirect }}=.016\right)$, which offsets the positive effect of grade homophily on the formation of triad structure. This can be demonstrated by summing the two single mediator indirect effects, which yields the joint indirect effect of students' grade $(-.003=-.019+.016)$. Since triangles cannot exist without two-stars, this result reveals that the total indirect effect of the GWESP pathway is in fact negative.

Results illustrate how omitting nesting structures can yield misleading results in ERGM. In Model 3, GWDSP is positive and significant, despite this effect being primarily driven by the higher-order GWESP term. Results also show how omitting nesting structure can complicate results from mediation analyses. If we were to not account for GWESP, we would be confronted with the unintuitive result that GWDSP and all homophily terms are positively related to tie probabilities, even though the indirect effect of grade homophily on GWDSP is negative. Researchers confronted with results such as these should recognize the strong possibility of omitted variables in their model. Moreover, if we were to not account for the effect of GWDSP, we would conclude that the mediating pathway is positive. However, since triangles cannot exist without two-stars, joint mediation analyses reveal that the joint indirect effect for the GWESP pathway is actually negative. Researchers should examine the joint mediating effect of lower-order terms when conducting mediation analyses with endogenous mediators. 


\subsection{Unobserved heterogeneity across levels of a moderator}

Unobserved heterogeneity across levels of a moderator is a known problem in nonlinear probability models. It is often discussed in terms of comparing coefficients across groups (Allison, 1999; Mood, 2010; Williams, 2009). Consider the simplest case of two groups with latent variable models representing the probability of realizing some outcome:

$$
\text { Group 0: } Y_{i j}^{* 0}=X^{0} \alpha^{0}+\tau^{0} \epsilon^{0} \quad \text { Group 1: } Y_{i j}^{* 1}=X^{1} \alpha^{1}+\tau^{1} \epsilon^{1}
$$

Since $\epsilon^{0}=\epsilon^{1}$, each model is linked to their respective nonlinear probability model through $\tau^{0}$ and $\tau^{1}$. The problem is that, in most cases, $\tau^{0} \neq \tau^{1}$. The differences in the observed coefficients thus cannot be interpreted as reflecting real differences in $\alpha^{0}$ and $\alpha^{1}$. Consequently, interaction coefficients can be biased and their $z$-statistics may be inaccurate (Allison, 1999; Mood, 2010). Even in scenarios when unobserved heterogeneity is not an issue, interactions have unclear meaning because coefficients for interaction terms in logistic-family models are not log-odds ratios, but rather the ratio of log-odds ratios for the main effects (Ai and Norton, 2003).

A number of strategies have been proposed to improve interpretation of interactions in nonlinear probability models (Allison, 1999; Kuha and Mills, 2018; Mustillo et al., 2012; Williams, 2009). Allison (1999) recommends estimating separate models for each level of an interaction and using likelihood ratios from each model to test the equivalence of the model parameters. The weakness of this approach is that it assumes that $\alpha^{0}=\alpha^{1}$, which is problematic in many settings. The test is also implausible in ERGM, since splitting network data into distinct samples will damage the representation of network structure. Williams (2009) recommends using a broader class of heterogenous choice models to deal with unobserved heterogeneity. However, these models assume independent observations, rendering them inappropriate for network analysis. Both Mood (2010) and Kuha and Mills (2018) suggest that these difficulties are a fundamental property of latent response models, 
and that researchers should endeavour to use continuous measures and linear models when possible. But graph theory assumes a meaningful difference between 0 and 1 to represent tie variables, and even generalizations of ERGM for valued outcomes map the weighted edge data to a binary ERGM reference distribution (Desmarais and Cranmer, 2012b; Krivitsky, 2012).

The approach taken here tests the significance of interactions using differences in marginal effects. Moderation exists when the effect of $X$ varies across levels of a second variable, $g$. We define the AME for a level of an interaction as $A M E_{\theta_{x}}^{g=k}$, where $\theta_{x}$ is the effect of interest and $g$ is the moderator with $k$ unique values. If $\theta_{x}$ is moderated by $g, A M E_{\theta_{x}}$ should be significantly different across $k$.

Following from Long and Mustillo's (2018) approach for evaluating interactions in nonlinear probability models, we test moderation by computing the second difference in $A M E_{\theta_{x}}$ :

$$
\triangle A M E_{\theta_{x}}^{g}=A M E_{\theta_{x}}^{g=k_{1}}-A M E_{\theta_{x}}^{g=k_{2}} .
$$

The second difference is the difference in $A M E_{\theta_{x}}$ between two levels of $g$. The null hypothesis is $H_{0}: \triangle A M E_{\theta_{x}}^{g}=0$. If the moderator is binary, $k 1$ and $k 2$ will always be 1 and 0 . If we are interested in comparisons between observed levels of an interaction, then we can specify $k 1$ and $k 2$ to be any observed values in the dataset. We can also set $k 1$ and $k 2$ to representative values or summary statistics, such as the mean plus or minus one standard deviation. The test statistic is a Wald test with Delta standard error:

$$
\sigma\left(\Delta A M E_{\theta_{x}}^{g}\right)=\sqrt{\operatorname{Var}\left(A M E_{\theta_{x}}^{g=k_{1}}\right)+\operatorname{Var}\left(A M E_{\theta_{x}}^{g=k_{2}}\right)-2 \operatorname{Cov}\left(A M E_{\theta_{x}}^{g=k_{1}}, A M E_{\theta_{x}}^{g=k_{2}}\right) .}
$$

Researchers may also want to report a single summary statistic for an interaction. A natural way to assess an interaction when $g$ is continuous is to calculate $\triangle A M E_{\theta_{x}}^{g}$ for all $k$ 
in increasing order. We define the average $\triangle A M E_{\theta_{x}}^{g}$ by

$$
\Delta A \bar{M} E_{\theta_{x}}^{g}=\frac{1}{N_{k}-1} \sum_{k-1} A M E_{\theta_{x}}^{g=k}-A M E_{\theta_{x}}^{g=k-1}
$$

where $N_{k}$ is the number of unique values in $g$. We can interpret $\Delta A \bar{M} E_{\theta_{x}}^{g}$ as the average change in $A M E_{\theta_{x}}$ between levels of $g$. We can also calculate the average absolute second difference if the interaction is curvilinear.

The significance of the interaction is tested with the average absolute Wald statistic for all $\triangle A M E_{\theta_{x}}^{g}$. We use the absolute value instead of the raw mean to account for curvilinear interactions, where the trendline of the interaction may be approximately zero, but $\triangle A M E_{\theta_{x}}^{g}$ is significant for most levels of $g$. The null hypothesis is $H_{0}:\left|\Delta A \bar{M} E_{\theta_{x}}^{g}\right|=0 .{ }^{10} \mathrm{~A}$ significant result means that, on average, $\triangle A M E_{\theta_{x}}^{g}$ is significantly different from zero.

Note that while the average absolute Wald statistic is a useful summary statistic, it should not be regarded as a replacement for second differences. If the mean absolute Wald statistic is insignificant, but at least one second difference is significant, we should not conclude that there is no moderation. Instead, we should conclude that $X$ is only moderated at certain levels of $g$.

\subsection{Example 3: Revisiting Birds of a Feather}

Examples 1 and 2 interpreted the coefficients for race homophily, sex homophily, and grade homophily directly. However, we cannot be sure whether the preference for similar-peer friendships is real or a consequence of unobserved heterogeneity. We now turn to friendship formation in Faux Dixon High to test moderating effects for grade and sex homophily. Faux Dixon High is a directed network of 248 friendship nominations among students in a high school. The network is simulated from one large high school in the National Longitudinal Study of Adolescent Health.

\footnotetext{
${ }^{10}$ Note that the average of a series of Wald tests follow an asymptotic standard normal distribution (Dumitrescu and Hurlin, 2012).
} 
Table 6: ERGM of friendships in Faux Dixon High.

\begin{tabular}{lc}
\hline Parameters & Model 5 \\
\hline Edges & $-3.55^{* * *}(.21)$ \\
Grade & $-.08^{*}(.04)$ \\
Sender & $.07(.04)$ \\
Receiver & $-.99^{* * *}(.04)$ \\
Absolute difference & \\
Sex (male is referent) & $.11(.07)$ \\
Sender & $.07(.07)$ \\
Receiver & $.24^{* * *}(.05)$ \\
Same sex & \\
Race (black is referent) & \\
Sender & $-.04(.17)$ \\
Latino & $.17^{*}(.07)$ \\
White & $.05(.16)$ \\
Other & \\
Receiver & $.29(.15)$ \\
Latino & $.26^{* * *}(.07)$ \\
White & $.25(.16)$ \\
Other & $3.07^{* * *}(.10)$ \\
Mutual & \\
\hline AIC & 9,608 \\
BIC & 9,735 \\
\hline$* * *<0.001,{ }^{* *} p<0.01,{ }^{*} p<0.05$. \\
\end{tabular}

Friendships in the network are modeled as a function of students' grade, sex, race, and the tendency for tie reciprocation. We include separate sender and receiver effects for grade, sex, and race because the network is directed. To assess grade and sex homophily, we include two interaction terms in the ERGM. Sex homophily term is equal to 1 if two students are the same sex and zero otherwise. Grade homophily is the absolute difference between two students' grades.

Table 6 presents results. Compared to black students, whites tend to nominate more friends and receive more frienship nominations. Latinos also tend to receive more friendship nominations than blacks. The parameter for mutual ties indicates that friendship ties are more likely to exist if they are reciprocal. The absolute difference in grade and same sex parameters are also significant. But, these parameters may be biased due to unobserved heterogeneity. 
Figure 3: Average marginal effects for students' sex, moderated by alters' sex. Red points are AMEs, bands are $95 \%$ confidence intervals.

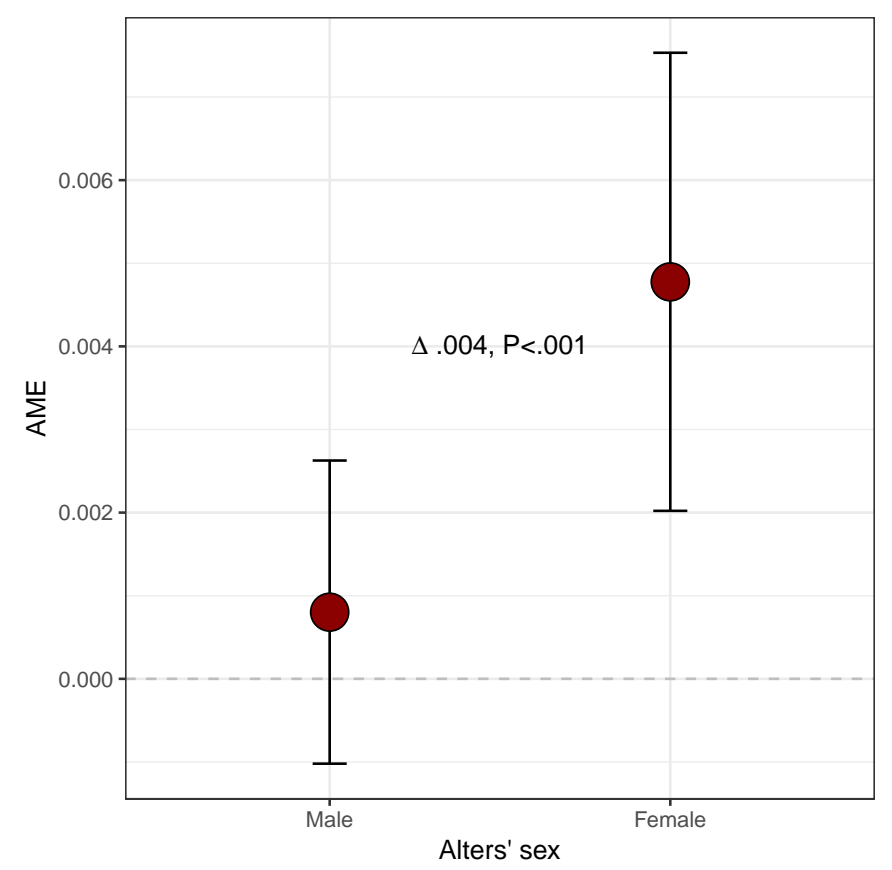

Figure 3 plots the AME by students' sex treating same sex friendships as a moderator. The AME for the female sender effect is insignificant when the receiving student is male, indicating that female students are no more likely to send friendship ties to male students than male students are to send friendship ties to anyone. In contrast, the AME for the female sender effect is significant when the receiving student is female, indicating that female students are $.5 \%$ more likely to nominate other female students as friends than male students are to nominate any other student as a friend $\left(A M E_{\text {female }}^{g=\text { female }}=.005\right)$. Since the main effect for female senders is insignificant in Model 5, we can be confident that this latter result is driven almost entirely be a preference for same-sex friendships. To provide a formal test, we calculate the second difference. The second difference is .004 ( $\mathrm{SE}=.001, p<.001)$, indicating that female-female friendships are $.4 \%$ more likely than female-male student friendships. This indicates a significant interaction for sex homophily.

Our second question is whether students tend to form friendships with students in similar grades. We assess this with the absolute difference between the senders' grade and the 
Figure 4: Average marginal effects for students' grade, moderated by alters'. Change scores are second differences.

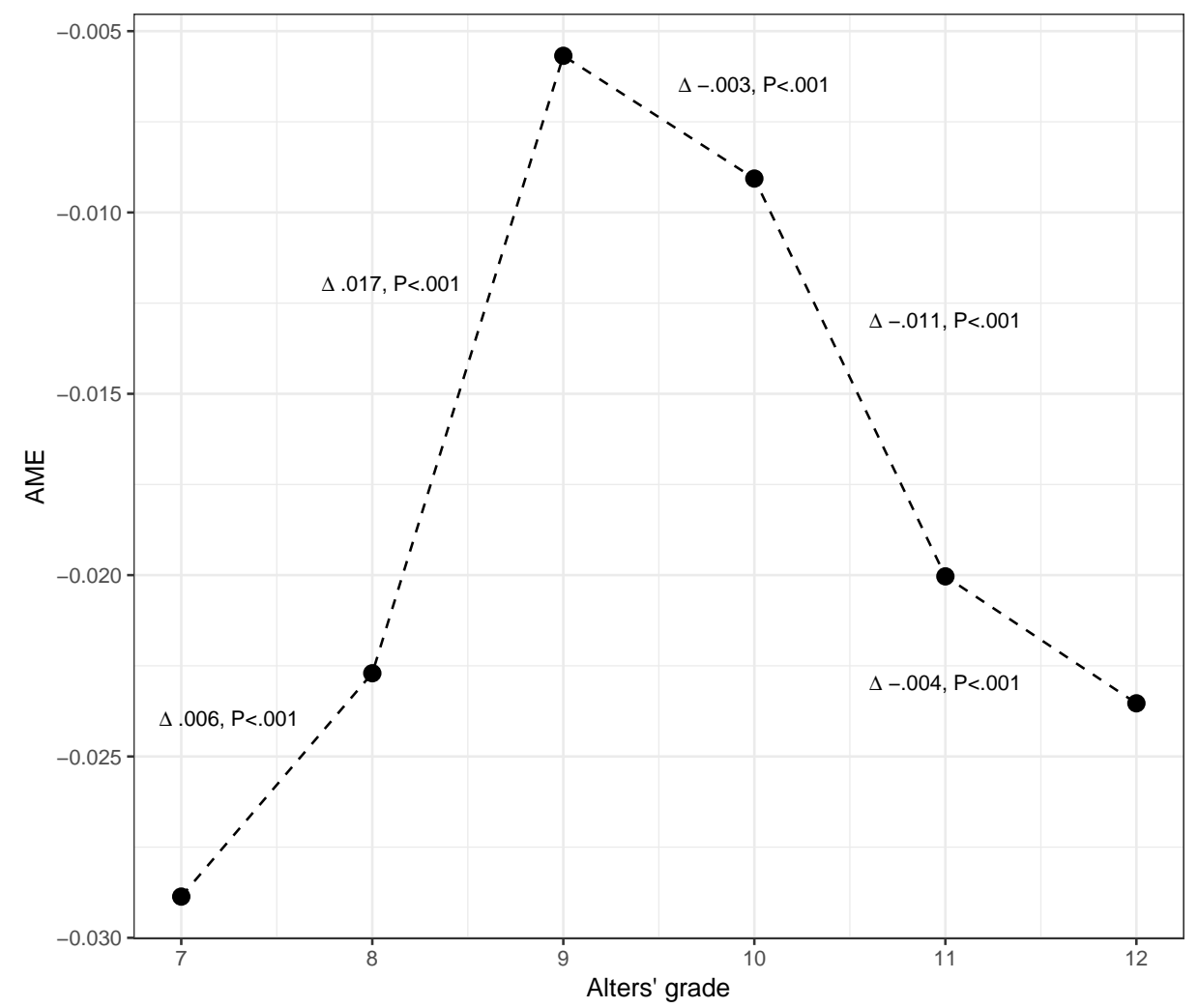

receivers' grade. To facilitate interpretation, we hold the senders' grade at its mean (9.39) when calculating the AME. ${ }^{11}$ Figure 4 shows an upsidedown $U$ shaped relationship, indicating that the AME is largest when the alter is in ninth grade, which is consistent with the homophily hypothesis. The second difference between grades 8 and 9 is $.017(p<.001)$, indicating that the AME for grade increases by .017 when the alter is in ninth grade instead of eighth grade.

In this setting, every second difference is statistically significant, which indicates a significant interaction. In other more ambiguous cases, we can also compute the mean second difference and mean absolute test statistic. The mean second difference is .001, which is

\footnotetext{
${ }^{11}$ The marginal effect for the interaction when we allow grade to vary is $\hat{p}_{i j}\left(1-\hat{p}_{i j}\right)\left(\theta_{\text {ego }}+\right.$ $\left.\theta_{\mid \text {difference } \mid}\left(\frac{X_{e g o}-g_{\text {alter }}=k}{\left|X_{\text {ego }}-g_{\text {alter }}=k\right|}\right)\right)$, which is the increase in tie probability when ego grade increases for an alter with a fixed grade. This interpretation is unintuitive because it does not tell us whether students prefer friendships when they are in similar grades. Holding ego grade at a constant value simplifies interpretation.
} 
consistent with the curvilinear trend in Figure 3. The mean absolute test statistic is 23.15 $(p<.001)$, which indicates that, on average, second differences are significantly different from zero. This reveals that the interaction is statistically significant.

An additional insight that can be gleaned from Figure 3 is the underlying mechanism contributing to sex and grade homophily. Researchers often interpret homophily as resulting from preferential attachment, though other possible mechanisms are possible (Schaefer et al., 2011; Snijders and Lomi, 2019). All of the AMEs in Figure 3 are negative, indicating that grade homophily never has a positive effect on tie probabilities. Instead, when two students are in a similar grade, the AME has the smallest negative effect on tie probabilities, indicating that students are least averse to forming friendships with other students when they are in similar grades. This is in contrast to sex homophily, where same-sex dyads increase the probability of friendship ties. These results suggest that grade homophily has less to do with a preference for peers in similar grades, and more to do with exposure, where students in dissimilar grades interact infrequently. In contrast, the positive AME for same sex friendships implies that same-sex friendship homophily is indeed a result of preferential attachment.

\subsection{Summary}

Unobserved heterogeneity problematizes comparisons between groups involved in ERGM interactions. Since many commonly used dyad covariates are interactions, this problem is especially salient in statistical network analyses. Methods based on marginal effects that correct for unobserved heterogeneity were proposed to assess moderation in statistical network models. Empirical applications demonstrated how the AME framework can be used to shed light on underlying mechanisms that contribute to homophilous friendship formation. 


\section{Moderated Mediation and Mediated Moderation}

Since unobserved heterogeneity can affect both moderation and mediation analyses, the case where a moderator is involved in a causal pathway requires special attention. There are two potential scenarios where moderators are involved in mediation: moderated mediation, when the mediator is part of an interaction, and mediated moderation, when a direct effect of interest is part of an interaction (Muller, Judd, and Yzerbyt, 2005).

\subsection{Moderated Mediation}

Moderated mediation occurs when the mediating effect of $M$ is moderated by a third variable (Preacher, Rucker, and Hayes, 2007). In some circumstances, a researcher will be interested in an interaction as a mediator; in others, researchers will simply be interested in the indirect effect of $M$ but wish to account for the potential that it is moderated by a third variable. Moderated mediation presents important difficulties when assessing mediation with the product of coefficients approach (see Mackinnon, 2008; Preacher et al., 2007). However, the problem is greatly simplified when using differences in AMEs.

The simplest case is when we are interested in whether an interaction coefficient mediates a direct effect. This reduces to a single-mediator model (Mackinnon, 2008), where we include the interaction coefficient and calculate the indirect effect as we would with any other single mediator analysis. In other circumstances, we are interested in assessing the total mediating effect of a variable and its interaction. In this case, we define $\mathbf{M}$ as a sequence of model terms including the interaction and its relevant main effects, $\mathbf{M}=M_{1}, M_{2}, \ldots M_{b}$. Regarded this way, the issue simplifies to joint mediation, where we include $\mathbf{M}$ into the full model and calculate the indirect effect. 


\subsection{Mediated Moderation}

A problem arises when $X$ is part of an interaction (Muller et al., 2005). If $X$ is part of an interaction, we cannot compute the indirect AME for $X$ as we would normally because $A M E_{\theta_{x}}$ and $A M E_{\theta_{x}^{p}}$ are moderated by $g$. We also cannot compare the coefficients for $X$ between models because these parameters may be biased by unobserved heterogeneity. There are three scenarios where we are interested in mediation when $X$ is part of an interaction: (1) when we want to assess whether $M$ mediates a main effect, net of the interaction, (2) when we want to assess whether $M$ mediates the interaction, net of the main effect, and (3) when we want to assess whether $M$ mediates the composite effect of the interaction and the main effect.

The first scenario is fairly simple to handle. If we are interested in whether $M$ mediates the main effect of an interaction, we compute the indirect AME for main effect of $X$, which is equivalent to fixing the interaction to zero. This gives us the change in AME if $g$ were to play no role.

The second scenario is more complicated because we need to isolate the effect of the moderator, net of the main effect. Defining the moderated effect as $\triangle A M E_{\theta_{x}}^{g}$, we can assess mediation by computing the difference-in-second difference, or the third difference $\Delta^{3} A M E_{\theta_{x}}^{g}$. When $X$ and $g$ are binary, there is only one second difference for the interaction. Thus, we can compute $\Delta^{3} A M E_{\theta_{x}}^{g}$ as:

$$
\Delta^{3} A M E_{\theta_{x}}^{g}=\Delta A M E_{\theta_{x}}^{g}-\Delta A M E_{\theta_{x}^{p}}^{g},
$$

with standard error:

$$
\sigma\left(\Delta^{3} A M E_{\theta_{x}}^{g}\right)=\sqrt{\operatorname{Var}\left(\Delta A M E_{\theta_{x}}^{g}\right)+\operatorname{Var}\left(\Delta A M E_{\theta_{x}^{p}}^{g}\right)-2 \operatorname{Cov}\left(\Delta A M E_{\theta_{x}}^{g}, \Delta A M E_{\theta_{x}^{p}}^{g}\right)},
$$

and covariance: $\operatorname{Corr}\left(\Delta M E_{\theta_{x}}^{g, i j}, \Delta M E_{\theta_{x}^{p}}^{g, i j}\right) \sigma\left(\Delta A M E_{\theta_{x}}^{g}\right) \sigma\left(\Delta A M E_{\theta_{x}^{p}}^{g}\right)$, which yields a Sobel- 
type test for the third difference. We can interpret $\triangle^{3} A M E_{\theta_{x}}^{g}$ as the difference in change in tie probability when $g$ moves from 0 to 1 . This interpretation is intuitive and substantively meaningful, but will often be cumbersome to communicate verbally. Typically graphical representations will be appealing (examples provided below). The total second difference is $\triangle A M E_{\theta_{x}}^{g}=\triangle A M E_{\theta_{x}^{p}}^{g}+\Delta^{3} A M E^{g}$ and the proportion mediated is $1-\frac{\triangle A M E_{\theta_{x}^{p}}^{g}}{\Delta A M E_{\theta_{x}}^{g}}$.

We can extend the mediation analysis to continuous moderators by computing $\Delta^{3} A M E_{\theta_{x}}^{g}$ for all pairs of second differences. As in moderation analyses, we can specify $g$ to be fixed at observed values, representative values, or measures of central tendency when calculating $\Delta^{3} A M E_{\theta_{x}}^{g}$. We can also compute the mean third difference or mean absolute third difference to consider whether, on average, there is a statistically significant indirect effect across levels of $g$. The test statistic is the mean absolute Wald statistic for the third differences.

We can take a similar approach to assess mediation for the composite effect of an interaction (the third scenario). The composite effect of an interaction is the $A M E_{\theta_{x}}^{g}$ at each level of $g$. Thus, to assess whether $M$ mediates the composite effect of an interaction, we compute the indirect AME for all levels of $g$ using Eq. (8). We can also average the indirect AME to yield the average mediated effect at all levels of the moderator. This approach is equivalent to the second scenario, except we conduct a Sobel-type test for the estimates of $A M E_{\theta_{x}}^{g}$ instead of $\triangle A M E_{\theta_{x}}^{g}$.

\subsection{Example 4: Concluding Birds of a Feather or Friend of a Friend}

Examples 1 and 2 treated node-matching as a main effect, instead of an interaction, in mediation analyses. We now revisit the mediation pathway for the indirect effects of grade and sex homophily in Faux Dixon High using a mediated moderation analysis that correctly casts homophily parameters as interactions. Table 7 presents results from an ERGM including the mediating variables GWDSP and GWESP to Model 5. The GWESP coefficient is significant and positive, while GWDSP is insignificant. 
Table 7: ERGM of friendships in Faux Dixon High.

\begin{tabular}{lc}
\hline Parameters & Model 6 \\
\hline Edges & $-4.82^{* * *}(.40)$ \\
Grade & $-.02(.05)$ \\
Sender & $.05(.05)$ \\
Receiver & $-.65^{* * *}(.05)$ \\
Absolute difference & \\
Sex (male is referent) & $.06(.10)$ \\
Sender & $.07(.10)$ \\
Receiver & $.08(.10)$ \\
Same sex & \\
Race (black is referent) & \\
Sender & $-.19(.26)$ \\
Latino & $.33^{* *}(.12)$ \\
White & $.09(.26)$ \\
Other & \\
Receiver & $-.21(.28)$ \\
Latino & $.12(.10)$ \\
White & $.21(.25)$ \\
Other & $1.86^{* * *}(.15)$ \\
Mutual & $-.02(.01)$ \\
GWDSP & $1.49^{* * *}(.08)$ \\
GWESP & $-3,677$ \\
& $-3,532$ \\
\hline AIC & \\
BIC &
\end{tabular}


Figure 5: Composite indirect effect of sex. Change statistics are indirect AMEs.

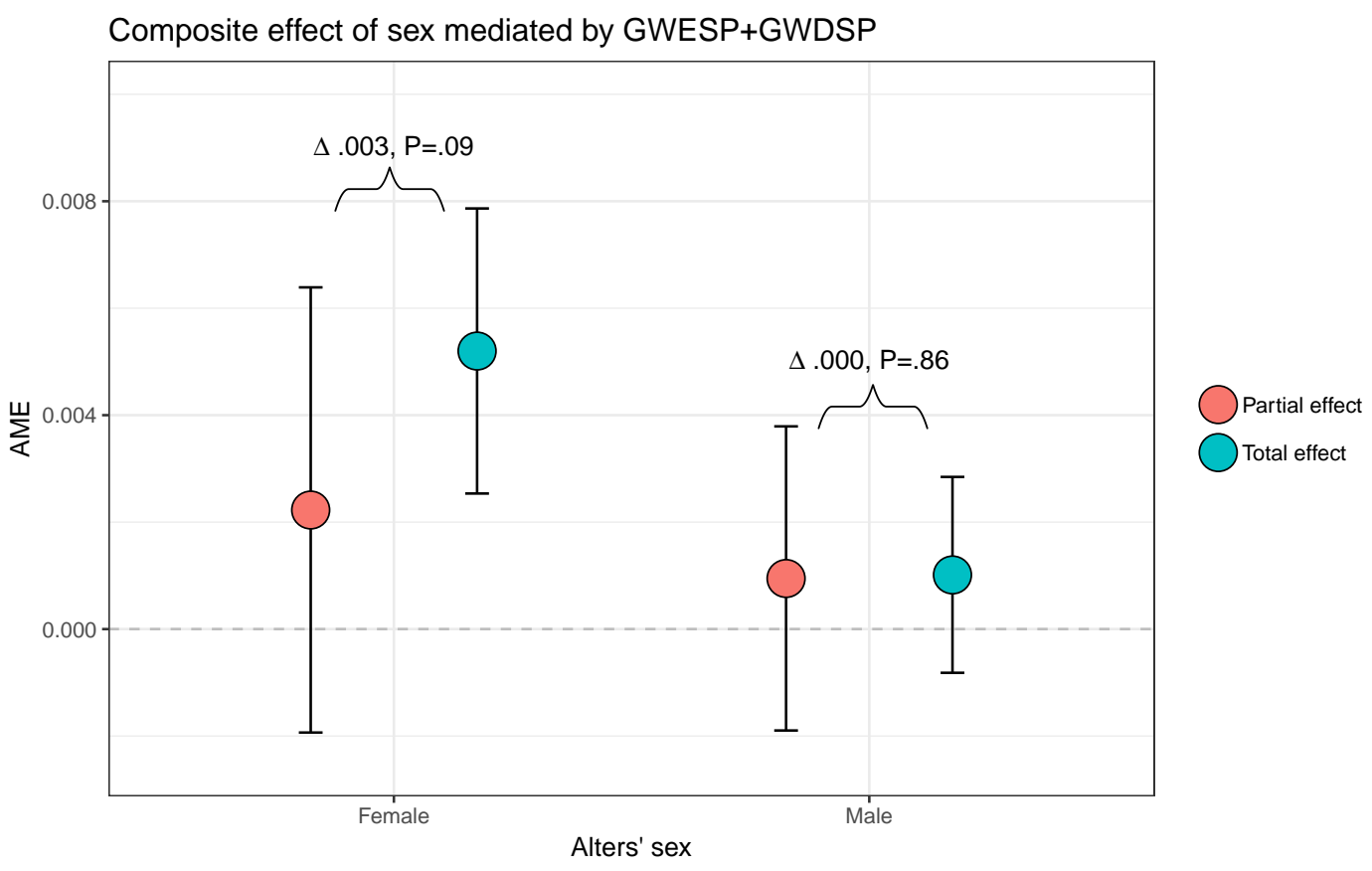

Figure 6: Mediated effect of sex homophily. Change statistics are third differences.

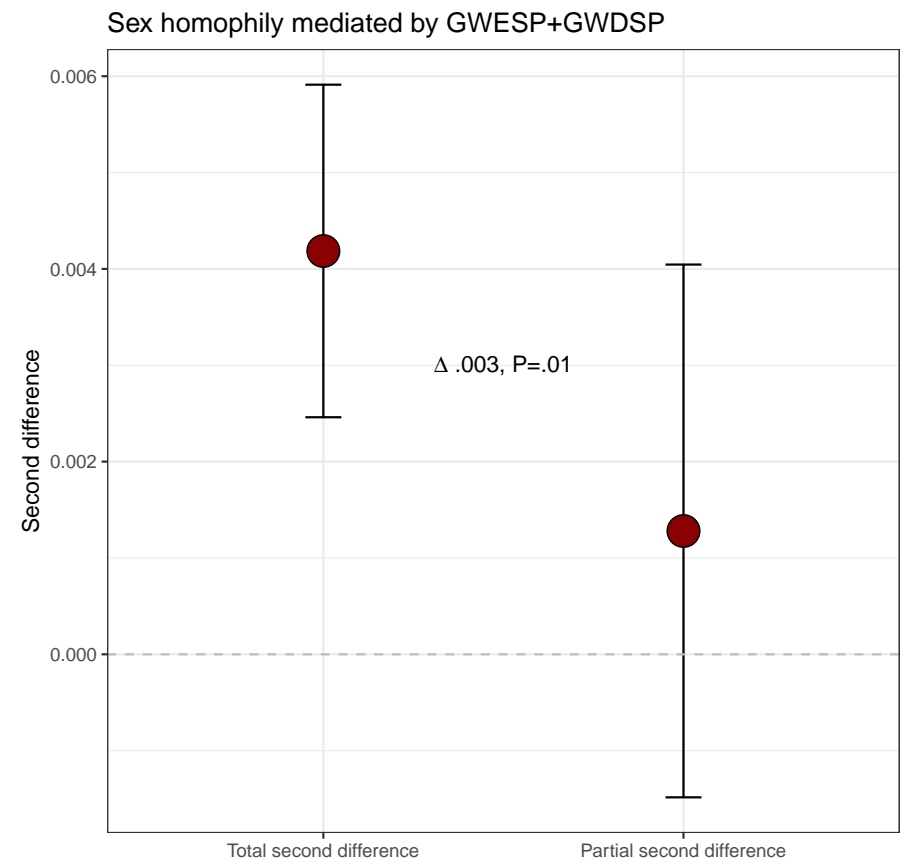


Our first question is whether GWESP mediates the composite effect of sex. Since GWDSP is nested in GWESP, we interpret the mediating effect of GWESP with respect to the joint pathway including both GWDSP and GWESP in a joint mediation analysis. The indirect effect for male alters is insignificant $\left(A M E_{\text {Female }}^{\text {indirect }, g=\text { Male }}=.000, p=.86\right)$, suggesting that GWESP does not mediate the main effect of sex (Figure 5). The indirect effect of sex for female alters is .003, but the effect is insignificant $\left(A M E_{\text {Female }}^{\text {indirect } g=\text { Female }}=.003, p=.09\right)$, suggesting that GWESP does not mediate the composite effect of sex.

The second question is whether GWESP mediates only the effect of same sex friendships, net of the main effect for students' sex. We assess this by computing the third difference (Figure 6). The third difference is significant, indicating that GWESP mediates the effect of sex homophily $\left(\Delta^{3} A M E_{\text {Female }}=.003, p=.01\right)$. Indeed, in Model 6 , the partial second difference for sex homophily is insignificant $\left(\triangle A M E_{\text {Female }}^{\text {partial }}=.001, p=.36\right.$. $)$, reflecting complete mediation. In total, being the same sex increases the probability of tie formation by $.4 \%\left(\triangle A M E_{\text {Female }}^{\text {total }}=.004\right)$, and roughly $75 \%$ of this increase can be attributed to triad formation (proportion mediated $=.73$ ). These results illustrate that while GWESP does not mediate the total effect of sex, it does mediate the effect of same sex friendships.

We conduct a similar analysis assessing students' grade. We again hold the senders' grade at its mean (9.39) when computing marginal effects for absolute differences. Figure 7 presents the composite mediated effect for students' grade. Complete results are presented in Table 8. The black nodes are the AMEs for the restricted model (Model 5), and the grey triangles are the AMEs for the full model (Model 6). The shaded grey area is the indirect effect. The indirect effect is significant at each unique value of grade. The mean proportion mediated is .43, indicating that, on average, GWESP mediates $43 \%$ of the effect of grade on tie probabilities. Indeed, the mean absolute Wald statistic is $7.13(p<.001)$, which indicates that the indirect effect is, on average, significant across levels of the interaction. The absolute size of the indirect AME is greatest when alters' grade is most dissimilar from senders' grade (e.g., alter in grade 7 or 12), reflecting that triad structure explains the largest 
Table 8: Mediating effect of GWESP and GWDSP on students' grade.

\begin{tabular}{lc}
\hline Grade & Indirect AME \\
\hline Sender grade (main effect) & $.000(.001)$ \\
Grade similarity (moderated effect) & \\
7 to 8 & $.003^{* * *}(.000)$ \\
8 to 9 & $.008^{* * *}(.001)$ \\
9 to 10 & $-.001^{* * *}(.000)$ \\
10 to 11 & $-.004^{* * *}(.001)$ \\
11 to 12 & $.000(.000)$ \\
Mean & .001 \\
Mean $\mid$ Wald $\mid$ & $7.528^{* * *}$ \\
Composite effect of grade & \\
7 & $-.014^{* * *}(.001)$ \\
8 & $-.011^{* * *}(.001)$ \\
9 & $-.003^{* * *}(.001)$ \\
10 & $-.004^{* * *}(.001)$ \\
11 & $-.008^{* * *}(.001)$ \\
12 & $-.008^{* * *}(.001)$ \\
Mean & -.008 \\
Mean $\mid$ Wald $\mid$ & $7.133^{* * *}$ \\
& \\
\hline
\end{tabular}

${ }^{* * *} p<0.001,{ }^{* *} p<0.01,{ }^{*} p<0.05$.

Figure 7: Composite indirect effect of grade. Black nodes are the partial model, grey triangles are the full model. Shaded area is the mediated effect.

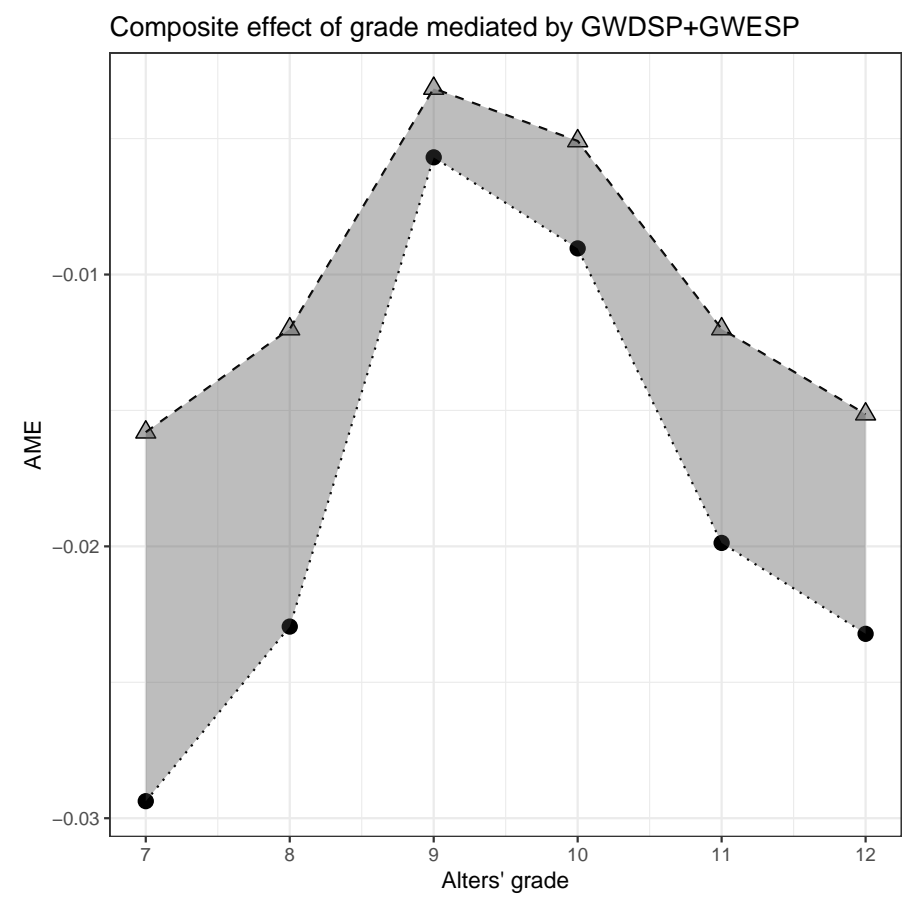


Figure 8: Indirect effect of grade homophily. Black nodes are the partial model, grey triangles are the full model. Shaded area is the mediated effect

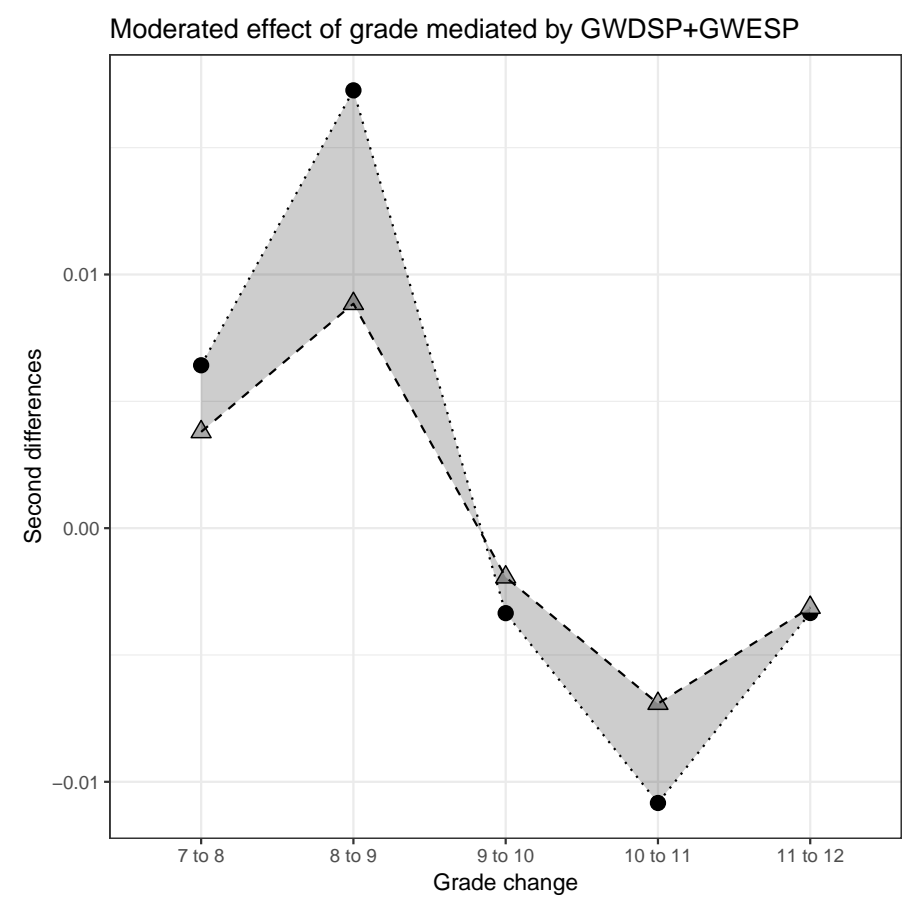

share of the composite effect of grade when students' grades are very different.

Next, we examine the third differences to assess whether GWESP mediates the effect of grade homophily. Figure 8 shows that the indirect effect is significant for most second differences, except for when alters' grade changes from 11 to 12 . The indirect effect also changes sign, from positive to negative, indicating that GWESP explains a declining portion of the effect of grade homophily as alters advance in grade. These results reveal nonlinearity in the indirect mediated effect depending on the value of the moderator. The mean absolute Wald statistic is 7.53, indicating that the indirect effect of grade homophily is, on average, statistically significant $(p<.001)$.

Collectively, results in Examples 1 through 4 shed light on how homophilous friendships and triadic structure constitute a pathway in school friendship networks. While GWESP does not mediate the main effect of either grade or sex, it does mediate a substantial portion of the effect of sex and grade homophily on friendship formation. These results replicate the substantive findings in Goodreau et al. (2009), though they also reveal how results 
may be complicated or confounded when the mediating pathway is improperly specified or moderation analyses do not correct for variation between groups.

\subsection{Summary}

Because unobserved heterogeneity problematizes both mediation and moderation analyses, special attention is required to assess mediation when moderators are direct effects or mediatiors. The difference in AME framework for moderation and mediation analyses integrates the two approaches in a straightforward fashion. These methods are especially important for interpreting mediating pathways involving homophily and heterophily.

\section{Discussion}

This study developed methods for mediation and moderation in statistical network models. All methods are based on post-estimation and can therefore be extended to any parametric network model with valid predictions, including SAOM (Snijders, 2001), relational event models (Butts, 2008), generalizations of ERGM (Desmarais and Cranmer, 2012b; Krivitsky, 2012), and temporal ERGM (Hanneke et al., 2010). These methods are implemented in the companion software package ergMargins for $\mathrm{R}$, freely available through the Comprehensive R Archive Network (CRAN).

Some network scholars have raised concern that greater attention needs to be given to interpreting tie probabilities (Desmarais and Cranmer, 2012a; Leifeld and Cranmer, 2019). Beyond mediation and moderation, marginal effects provide an appealing framework for interpreting coefficients in statistical network models (also see Mize, 2019). To do so, a researcher simply computes the AME for a coefficient. The resulting AME has a z-statistic equivalent to the parameter $z$-statistic, but can be interpreted on the scale of tie probabilties (e.g., a one unit increase in $X$ is associated with an $A M E_{\theta_{x}}$ increase in the probability of a tie forming). 
Marginal effects can also be used to improve the interpretation of structural effects. Since change in higher-order structures necessitates change in at least one lower-order structure, parameters for higher-order structural effects should, in principle, be interpreted with respect to lower-order effects. However, since tie probabilities are a multiplicative function of ERGM terms, this interpretation has been difficult and unintuitive within the framework of log-odds ratios. AMEs provide an additive framework to facilitate the interpretation of endogenous model parameters. For instance, because a triangle closes two two-stars, we can interpret the effect of closing a single triangle as an $A M E_{\text {triangle }}+2 A M E_{t w o-s t a r}$ increase in tie probability.

The mediation tests developed here can be construed as Sobel-type tests. Sobel tests have been historically critiqued for providing conservative standard errors in small sample spaces (Bollen and Stine, 1990; Mackinnon, 2008; Mackinnon et al., 2004). This is a relatively small concern for network anlaysis, since the dyad sample space grows exponentially with network size, and even small networks contain a large number of dyads. Even so, bootstrap standard errors can be easily obtained for the indirect effect in research examining very small networks. They are also an option if long runtimes are unconcerning or computing clusters are readily available. That said, bootstrap standard errors will typically be intractable for very large networks regardless of computing power and computation times will be restrictive in even moderately sized networks and high dimensional models.

One potential direction for future research is to develop computationally efficient methods for obtaining the standard error for the indirect effect. Though less intensive than other common variance estimators, like bootstrapping, a weakness of the Delta method is that it can be computationally demanding in high dimensional models or very large networks. Provisional experience indicates that Delta standard errors will rarely-if ever-take longer to compute than fitting an ERGM. However, as ERGMs are increasingly applied to large networks with hundreds of thousands of vertices (An, 2016; Goodreau, 2007), scalability becomes a larger concern. Potential resolutions include parallel estimation of Delta standard errors or asymptotic approximations using large subsamples of model predictions in very large 
networks.

Future research should also seek to develop causal mediation methods for statistical network models (Imai et al., 2010; Pearl, 2012; VanderWeele, 2011). Most causal mediation methods rely on knowledge of $\psi$, which problematizes their extension to statistical network models. Nevertheless, causal inference is of concern to network researchers (An, 2015), especially when funds are unavailable to experimentally manipulate network composition. Developing methods for causal mediation analyses will help improve understanding of causal pathways in research on network dynamics. 


\section{References}

Agresti, Alan. 2002. Categorical Data Analysis. New York: Wiley.

Ai, Chunrong and Edward C. Norton. 2003. "Interaction Terms in Logit and Probit Models." Economics Letters 80:123 - 129.

Allison, Paul D. 1999. "Comparing Logit and Probit Coefficients Across Groups." Sociological Methods and Research 28:186 - 208.

An, Weihua. 2015. "Multilevel Meta Network Analysis with Application to Study Network Dynamics of Network Interventions." Social Networks 43:48-56.

An, Weihua. 2016. "Fitting ERGMs on big networks." Social Science Research 59:107-119.

Baron, Reuben M. and David A. Kenny. 1986. "The Moderator-Mediator Variable Distinction in Social Psychological Research: Conceptual, Strategic, and Statistical Consideration." Journal of Personality and Social Psychology 51:1,173 - 1,182.

Barrett, Tyson S., Rick A. Cruz, and Ginger Lockhart. 2019. "Marginal Mediation Analysis: A Practical Statistical Framework for Interpretable Mediated Effects." Working paper. pp. $1-16$.

Besag, Julian E. 1972. "Nearest-Neighbour Systems and the Auto-Logistic Model for Binary Data." Journal of the Royal Statistical Society Series B 34:75 - 83.

Besag, Julian E. 1974. "Spatial Interaction and the Statistical Analysis of Lattice Systems." Journal of the Royal Statistical Society Series B 36:192 - 236.

Block, Per. 2015. "Reciprocity, transitivity, and the mysterious three-cycle." Social Networks 40:163-173.

Block, Per, Christoph Stadtfeld, and Tom A. B. Snijders. 2019. "Forms of Dependence: Comparing SAOMs and ERGMs From Basic Principles." Sociological Methods 83 Research 48:202-239.

Bollen, Kenneth A. and Robert Stine. 1990. "Direct and Indirect Effects: Classical and Bootstrap Estimates of Variability." Sociological Methodology 20:115 - 140.

Box-Steffensmeier, Janet M., Dino P. Christenson, and Jason W. Morgan. 2018. "Modeling 
Unobserved Heterogeneity in Social Networks with the Frailty Exponential Random Graph Model." Political Analysis 26:3 - 19.

Breen, Richard, Kristian Bernt Karlson, and Anders Holm. 2013. "Total, Direct, and Indirect Effects in Logit and Probit Models." Sociological Methods and Research 42:164-191.

Butts, Carter T. 2008. "A Relational Event Framework for Social Action." Sociological Methodology 38:155 -200.

Coleman, James. 1990. Foundations of Social Theory. Belnap Press.

Cramer, Jan Salomon. 2007. "Robustness of Logit Analysis: Analysis for Unobserved Heterogeneity and Mis-specified Disturbances." Oxford Bulletin of Economics and Statistics 69:545-555.

Desmarais, Bruce A. and Skyler J. Cranmer. 2012a. "Micro-level Interpretation of Exponential Random Graph Models with Application to Estuary Networks." Policy Study Journals 40:402 -431.

Desmarais, Bruce A. and Skyler J. Cranmer. 2012b. "Statistical Inference for Valued-Edge Networks: The Generalized Exponential Random Graph Model.” PLoS One .

Dumitrescu, Elena Ivona and Christophe Hurlin. 2012. "Testing for Granger Non-causality in Heterogenous Panels." Economics Modeling 29:1,450 - 1,460.

Duxbury, Scott. 2018. "Diagnosing Multicollinearity in Exponential Random Graph Models." Sociological Methods and Research DOI: 10.1177/0049124118782543.

Erdos, Paul and Alfred Renyi. 1959. "On Random Graphs." Publicationes Mathematicae $6: 290-297$.

Faust, Katherine. 2007. "Very Local Structure in Social Networks." Sociological Methodology $37: 209-256$.

Frank, Ove and David Strauss. 1986. "Markov Graphs." Journal of the American Statistical Association 81:832 - 842.

Gelman, Andrew and Hal Stern. 2006. "The Difference Between "Significant" and "Not Significant" is not Itself Statistically Significant." The American Statistician 60:328 - 
331.

Geyer, Charles J. and Elizabeth A. Thompson. 1992. "Constrained Monte Carlo Maximum Likelihood for Dependent Data." Journal of the Royal Statistical Society B 54:657 699.

Goodreau, Steven M. 2007. "Advances in Exponential Random Graph (p*) Models Applied to a Large Social Network." Social Networks 29:231-248.

Goodreau, Steven M., James A. Kitts, and Martina Morris. 2009. "Birds of a feather, or friend of a friend? Using exponential random graph models to investigate adolescent social networks." Demography 46:103-125.

Granovetter, Mark S. 1973. "The strength of weak ties." American Journal of Sociology 78:347-367.

Handcock, Mark S., David R. Hunter, Carter T. Butts, Steven M. Goodreau, and Martina Morris. 2008. "statnet: Software Tools for the Representation, Visualization, Analysis and Simulation of Network Data." Journal of Statistical Software 24:1 -19.

Hanneke, Steve, Wenjie Fu, and Eric P. Xing. 2010. "Discrete Temporal Models of Social Networks." Electronic Journal of Statistics 4:585 - 605.

Hayes, Andrew F. and Kristopher J. Preacher. 2010. "Quantifying and Testing Indirect Effects in Simple Mediation Models When the Constituent Paths are Nonlinear." Multivariate Behavioral Research 45:627 - 660.

Hinton, G. E. and R. R. Salakhutdinov. 2006. "Reducing the Dimensionality of Data with Neural Networks." Science 313:504-507.

Holland, Paul W. and Samuel Leinhardt. 1981. "An Exponential Family of Probability Distributions for Directed Graphs." Journal of the American Statistical Association $76: 33-50$.

Hosmer, David W. and Stanley Lemeshow. 2000. Applied Logistic Regression. WileyInterscience Publication.

Hunter, David R. 2007. "Curved Exponential Family Models for Social Networks." Social 
Networks 29:216 - 230 .

Imai, Kosuke, Dustin Tingley, and Luke Keele. 2010. "A General Approach to Causal Mediation Analysis." Psychological Methods 15:309 - 334.

Judd, Charles M. and David A. Kenny. 1981. "Process Analysis: Estimating Mediation in Treatment Evaluations." Evaluation Review 5:602 - 619.

Karlson, Kristian Bernt, Anders Holm, and Richard Breen. 2012. "Comparing Regression Coefficients Between Same-sample Nested Models Using Logit and Probit: A New Method." Sociological Methodology 42:286 - 313.

Krivitsky, Pavel. 2017. "Using Contrastive Divergence to Seed Monte Carlo MLE for Exponential-family Random Graph Models." Computational Statistics and Data Analysis 107:149 -161.

Krivitsky, Pavel N. 2012. "Exponential-family Random Graph Models for Valued Networks." Electronic Journal of Statistics 6:1,100 - 1,128.

Kuha, Jouni and Colin Mills. 2018. "On Group Comparisons with Logistic Regression Models." Sociological Methods and Research .

Leifeld, Philip and Skylar Cranmer. 2019. "A Performance Based Comparison of the Temporal Exponential Random Graph Model and Stochastic Actor Oriented Model." Network Science 7:20 - 51.

Lewis, Kevin. 2013. "The Limits of Racial Prejudice." Proceedings of the National Academy of Sciences 110:18,814-18,819.

Lewis, Kevin and Jason Kaufman. 2018. "The Conversion of Cultural Tastes into Social Network Ties." American Journal of Sociology 123:1684-1742.

Long, J. Scott. 1997. Regression Models for Categorical and Limited Dependent Variables. Thousan Oaks, CA: SAGE Publications.

Long, J. Scott and Sarah A. Mustillo. 2018. "Using Predictions and Marginal Effects to Compare Groups in Regression Models for Binary Outcomes." Sociological Methods and Research . 
Lusher, Dean, Johan Koskinen, and Garry Robins. 2013. Exponential Random Graph Models for Social Networks. UK: Cambridge University Press.

Mackinnon, David P. 2008. Introduction to Statistical Mediation Analysis. Routledge.

Mackinnon, David P. and James H. Dwyer. 1993. "Estimating Mediated Effects in Prevention Studies." Evaluation Review 17:144-158.

Mackinnon, David P., Chondra M. Lockwood, Hendricks Brown, and Wei Wang. 2007. "The Intermediate Endpoint Effect in Logistic and Probit Regression." Clinical Trials 4:499 $-513$.

Mackinnon, David P., Chondra M. Lockwood, and Jason Williams. 2004. "Confidence Limits for the Indirect Effects: Distribution of the Product and Resampling Methods." Multivariate Behavioral Research 39:99 - 128.

Mackinnon, David P., Ghulam Warsi, and James H. Dwyer. 1995. "A Simulation Study of Mediated Effect Measures." Multivariate Behavioral Research 30:41 - 62.

McPherson, Miller, Lynn Smith-Lovin, and James M. Cook. 2001. "Birds of a Feather: Homophily in Social Networks." Annual Review of Sociology 27:415 - 444.

Melamed, David, Ashely Harrell, and Brent Simpson. 2018. "Cooperation, Clustering, and Assortative Mixing in Dynamic Networks." Proceedings of the National Academy of Sciences 115:951-956.

Mize, Trenton D. 2019. "Best Practices for Estimating, Interpreting, and Presenting Nonlinear Interaction Effects." Sociological Science .

Mood, Carina. 2010. "Logistic Regression: Why We Cannot Do What We Think We Can Do, and What We Can Do About It." European Sociological Review 26:67 - 82.

Morris, Martina, Mark S. Handcock, and David R. Hunter. 2008. "Specification of Exponential- Family Random Graph Models: Terms and Computational Aspects." Journal of Statistical Software 24:1 -22.

Muller, Domnique, Charles M. Judd, and Vincent Y. Yzerbyt. 2005. "When Moderation is Mediated and Mediation is Moderated." Journal of Personality and Social Psychology 
$89: 852-863$.

Mustillo, Sarah, Lawrence R. Landerman, and Kenneth C. Land. 2012. "Modeling Longitudinal Count Data: Testing for Group Differences in Growth Trajectories Using Average Marginal Effects." Sociological Methods and Research 41:467 - 487.

Newman, Mark E.J. 2010. Networks: An Introduction. Oxford University Press.

Papachristos, Andrew V. and Sara Bastomski. 2018. "Connected in Crime: The Enduring Effect of Neighborhood Networks on the Spatial Patterning of Violence." American Journal of Sociology 124:517-568.

Papachristos, Andrew V., David M. Hureau, and Anthony A. Braga. 2013. "The Corner and the Crew: The Influence of Geography and Social Networks on Gang Violence." American Sociological Review 78:417 - 447.

Papachristos, Andrew V., Christopher Wildeman, and Elizabeth Roberto. 2015. "Tragic, but not Random: The Social Contagion of Nonfatal Gunshot Injuries." Social Science and Medicine 125:1-7.

Pearl, Judith. 2012. "The Causal Mediation Formula-A Guide to the Assessment of Pathways and Mechanisms." Prevention Science 13:426 - 436.

Preacher, Kristopher J. and Andrew F. Hayes. 2008. "Asymptotic and Resampling Strategies for Assessing and Comparing Indirect Effects in Multiple Mediator Models." Behavioral Research Methods 40:879 - 891.

Preacher, Kristoph J., D. D. Rucker, and Andrew F. Hayes. 2007. "Addressing Moderated Mediation Hypotheses: Theory, Methods, and Prescriptions." Multivariate Behavioral Research 42:185 - 227.

Schaefer, David R., Olga Kornienko, and Andrew M. Fox. 2011. "Misery Does Not Love Company: Network Selection Mechanisms and Depression Homophily." American Sociological Review 76:764-785.

Snijders, Tom A.B., Phillipa E. Pattison, Garry L. Robins, and Mark S. Handcock. 2006. "New Specifications for Exponential Random Graph Models." Sociological Methodology 
36:99- 150.

Snijders, Tom A. B. 1996. "Stochastic actor-oriented models for network change." Journal of Mathematical Sociology 21:149 - 172.

Snijders, Tom A. B. 2001. "The Statistical Evaluation of Social Network Dynamics." Sociological Methodology 31:361-395.

Snijders, Tom A. B. 2002. "Markov Chain Monte Carlo Estimation of Exponential Random Graph Models." Journal of Social Structure 3.

Snijders, Tom A. B. 2011. "Statistical Models for Social Networks." Annual Review of Sociology 37:131-153.

Snijders, Tom A. B. and Alessandro Lomi. 2019. "Beyond Homophily: Incorporating Actor Variables in Statistical Network Models." Network Science 7:1 -19.

Sobel, Michael E. 1982. "Asymptotic Confidence Intervals for Indirect Effects in Structural Equation Models." Sociological Methodology 13:290-312.

Sobel, Michael E. 1986. "Direct and Indirect Effects in Linear Structural Equation Models." Sociological Methods and Research 16:155 - 176.

Strauss, David and Michael Ikeda. 1990. "Pseudolikelihood Estimation for Social Networks." Journal of the American Statistical Association 85:204 - 212.

van Duijn, Marijtje A., Krista J. Gile, and Mark S. Handcock. 2009. "A Framework for the Comparison of Maximum Pseudolikelihood and Maximum Likelihood Estimation of Exponential Family Random Graph Models." Social Networks 31:52 - 62.

van Duijn, Marijtje A., Tom A. B. Snijders, and Bonne J. H. Zijlstra. 2004. "p2: a Random Effects Model with Covariates for Directed Graphs." Statistica Neerlandica 58:234 254.

VanderWeele, Tyler J. 2011. "Causal Mediation Analysis with Survival Data." Epidemiology $22: 575-581$.

VanderWeele, Tyler J. and Stijn Vansteelandt. 2013. "Mediation Analysis with Multiple Mediators." Epidemiologic Methods 2:95 - 115. 
Wasserman, Stan and Phillipa Patterson. 1996. "Logit Models and Logistic Regressions for Social Networks I: An Introduction to Markov Graphs and p*." Psychometrika 61:401 -425 .

Watts, Duncan J. and Steven H. Strogatz. 1998. "Collective dynamics of 'small-world' networks." Nature 393:440 - 442.

Williams, Richard. 2009. "Using Heterogeneous Choice Models to Compare Logit and Probit Coefficients across Groups." Sociological Methods and Research 37:531 - 559 .

Wimmer, Andreas and Kevin Lewis. 2010. "Beyond and below racial homophily: ERG models of a friendship network documented on Facebook." American Journal of Sociology 2:583-642.

Winship, Christopher and Robert D. Mare. 1983. "Structural Equations and Path Analysis for Discrete Data." American Journal of Sociology 89:54 - 110.

Wooldridge, Jeff. 2002. Econometric Analysis of Cross Section and Panel Data. MIT Press. 